\title{
Magnoliophyta, Arly National Park, Tapoa, Burkina Faso
}

\author{
Oumarou Ouédraogo ${ }^{1}$, Marco Schmidt ${ }^{2,3,4^{*}}$, Adjima Thiombiano ${ }^{1}$, Karen Hahn ${ }^{3,4}$, Sita Guinko ${ }^{1}$ and \\ Georg Zizka 2,3, 4 \\ 1 Université de Ouagadougou, Laboratoire de Biologie et Ecologie Végétales, UFR/SVT. 0309 B.P. 848 Ouagadougou 09, Burkina Faso. \\ 2 Senckenberg Research Institute, Department of Botany and molecular Evolution. Senckenberganlage 25, 60325. Frankfurt am Main, Germany \\ 3 J.W. Goethe-University, Institute for Ecology, Evolution \& Diversity. Siesmayerstr. 70, 60054. Frankfurt am Main, Germany \\ 4 Biodiversity and Climate Research Institute (BiK-F), Senckenberganlage 25, 60325. Frankfurt am Main, Germany. \\ * Corresponding author. E-mail: marco.schmidt@senckenberg.de
}

\begin{abstract}
The Arly National Park of southeastern Burkina Faso is in the center of the WAP complex, the largest continuous system of protected areas in West Africa. Although well known for its large mammal populations, its flora has largely been unexplored until recently. The plant species composition is typical for sudanian savanna areas with a high share of grasses and legumes and similar to other protected areas of the complex, the neighbouring Pama reserve and W National Park. It has more species in common with the classified forest of Kou in SW Burkina Faso than with the geographically closer Sahel reserve. The 490 species belong to 280 genera and 83 families. The most important life forms are phanerophytes and therophytes.
\end{abstract}

\section{INTRODUCTION}

For Burkina Faso, only very few comprehensive assessments of local floras have been published (e.g., Guinko and Thiombiano 2005; Ouoba et al. 2006; Gnoumou et al. 2008). There is virtually no information available about the flora of the country's protected areas, although this knowledge is important for the conservation of endangered species and habitats and the management of these protected areas. Even on the larger scale of West Africa, local assessments of the flora are mainly restricted to the forest region (Jongkind et al. 1999; Jongkind 2007a, b; Aké Assi et al. 2005; Ekpe 2005; Holié and Delamou 2004; 2006; Couch and Williams 2006; Luke 2007; Siaw and Dabo 2007). Following a recent publication on the partial faunal reserve of Pama (Mbayngone et al. 2008) this paper continues to close gaps in knowledge on the plant diversity of West African protected areas by providing a comprehensive checklist of Arly National Park.

\section{MATERIALS AND MethodS \\ Study Area}

The Arly National Park (Figure 1) resulted from the merging of the total reserve of Madjoari and the total reserve of Arly in 1978 (Ouattara 1994) and is presently a protected area of IUCN category II. The forestry authorities have further increased the area to include a part of the Gobnangou mountain chain. The park covers now an area of 120.000 ha and is part of the so-called WAP complex (W-Arly-Pendjari), a large transfrontier complex of nature reserves consisting of Arly National Park, the neighbouring Pendjari National Park south of the Pendjari river in Benin, the W National Park shared by Benin, Burkina Faso and Niger, several partial faunal reserves and hunting zones. Hunting is forbidden in Arly National Park and by lack of infrastructure there is only limited ecotourism possible at present. The whole complex is well-known for its wildlife including large populations of elephants and lions (Lamarque 2004; Balança et al. 2007). It has a much denser vegetation than the surrounding areas, where agriculture has encroached on savannas and forests and tall perennial grasses almost disappeared, so that its borders are even visible from space (e.g. in the map in UNEP 2008).

Among the reserves of the WAP complex, Arly has a high habitat diversity, including extensive gallery forests of the Arly and Pendjari rivers (Figure 2), sandstone hills of the Gobnangou chain (Figure 3), sudanian savannas dominated by tall perennial grasses and closed woodlands.

The park has been inventoried for birds (Green and Sayer 1979) and large mammals (Green 1979) but detailed plant information is up to now restricted on studies on woody plant structure (Ouédraogo et al. 2008) and regeneration (Ouédraogo et al. 2009).

\section{Data collection}

The species listed here were compiled mainly from the surveys by O. Ouédraogo in the years 2004 to 2007 covering all habitat types of the park, complemented by several herb layer relevés conducted by M. Schmidt in

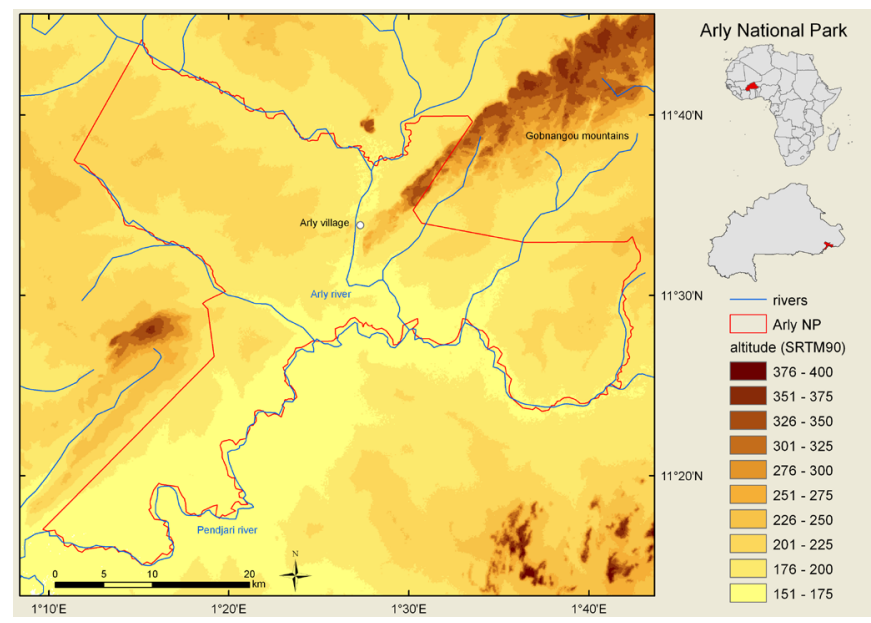

Figure 1. Map of Arly National Park with boundary and watercourses. Altitudinal information is taken from SRTM 90 data. 
2002 (Schmidt 2006), rapid assessments of an expedition in late 2008 and specimen data from the herbarium of the University of Ouagadougou (OUA) and the Herbarium Senckenbergianum (FR). The species names and synonymy follow the African Flowering Plants Database (Gautier et al. 2006), families are assigned according to Brummit (1992), but family names like Compositae or Gramineae are replaced by the standard names referring to the type genus. Species have been determined using Hutchinson and Dalziel (1954-1972), Berhaut (1971-1988), Arbonnier (2002), Akoègninou et al. (2006) and the West African Plants Database (http://westafricanplants.senckenberg. de, Brunken et al. 2008).

Life form information has been assembled from Guinko (1984), Aké Assi (2001; 2002) and supplemented by own observations. The more detailed information in the mentioned literature has been reduced to the main types, as defined by Raunkiaer (1905), in order to have a comparable definition of life forms.

The chorological types refer to the phytochoria of White (1983), also used in other studies of the region (Sinsin 2001; Mbayngone et al. 2008).

\section{Data analysis}

The comparisons with other reserves in Burkina Faso are based on the publications of Guinko and Thiombiano (2005) for the Forêt Classée de la Kou, Mbayngone et al. (2008) for the Reserve partielle de la faune de Pama, Schmidt et al. (2008) for the Reserve sylvo-pastorale et partielle de faune du Sahel and Arbonnier et al. (2002) for the Niger part of the Parc National de la $W$ du Niger. The nomenclature has been harmonized using the synonymy of the African Flowering Plants Database (Gautier et al. 2006), therefore slight differences from the original publications are possible. Calculation of species in common and exclusive to certain reserves has been done in Microsoft Excel, calculation of Sørensen indices and clustering has been done in Community Analysis Package (v.4, Pisces Conservation Ltd.).

\section{RESULTS AND DISCUSSION}

The Arly National Park contributes highly to the conservation of the country's flora. We found 490 species (Table 1) which represent about $30 \%$ of all vascular plants inventoried in Burkina Faso (Schmidt et al. 2010). The Park's flora consists of 280 genera belonging to 83 families. The ten most species rich families are Fabaceae (95 species), followed by Poaceae (88), Cyperaceae (27), Rubiaceae (22), Combretaceae (19), Euphorbiaceae (14), Acanthaceae (13), Convolvulaceae (13), Malvaceae (12) and Asteraceae (11) (Figure 4).

The family composition (Figure 4) is very similar to the family composition of the nearby Pama reserve (Mbayngone et al. 2008). The most striking difference is the higher family richness in Arly (83 as compared to 73) and the higher species richness in some families, especially the Combretaceae.

Similar to the family composition of the Pama reserve and W National Park, the dominance of grasses and legumes in Arly National Park is very typical of savannas (Bourlière and Hadley 1970) and is found throughout Burkina Faso (Schmidt et al. 2005; Schmidt et al. 2010), up to the very North (Schmidt et al. 2008) and in the nearby Atakora mountains of Benin (Wala unpublished data).

The ten most species rich genera are Indigofera (18 species), Combretum (11), Cyperus (11), Ipomoea (9), Acacia (8), Crotalaria (8), Andropogon (7), Eragrostis (7), Hyparrhenia (7) and Tephrosia (7). Altogether they constitute $19 \%$ of the National Park's flora.

The life form composition (Figure 5) is dominated by phanerophytes (40\%) and therophytes (33\%), followed by chamaephytes (10\%), hemicryptophytes (9\%), geophytes (5\%), hydrophytes (2\%) and helophytes (1\%). This is a typical pattern for the North Sudanian Zone (Hahn-Hadjali et al. 2006). Towards the Sahel the portion of therophytes increases at the expense of the phanerophytes (Schmidt et al. 2005). In the South Sudanian Zone phanerophytes are even more important (Koulibaly et al. 2006).

The chorological spectrum of the flora (Figure 6) is characterized by a high proportion of species specific to savanna regions (sudanian, sudano-zambesian) and a high proportion of wide-spread species (pantropical, paleotropical, pluriregional african, afrotropical). Sudanoguinean and guineo-congolian elements are mainly confined to the gallery forests.

The comparison of published inventories of protected areas of the region (Figure 7, Table 1) includes two

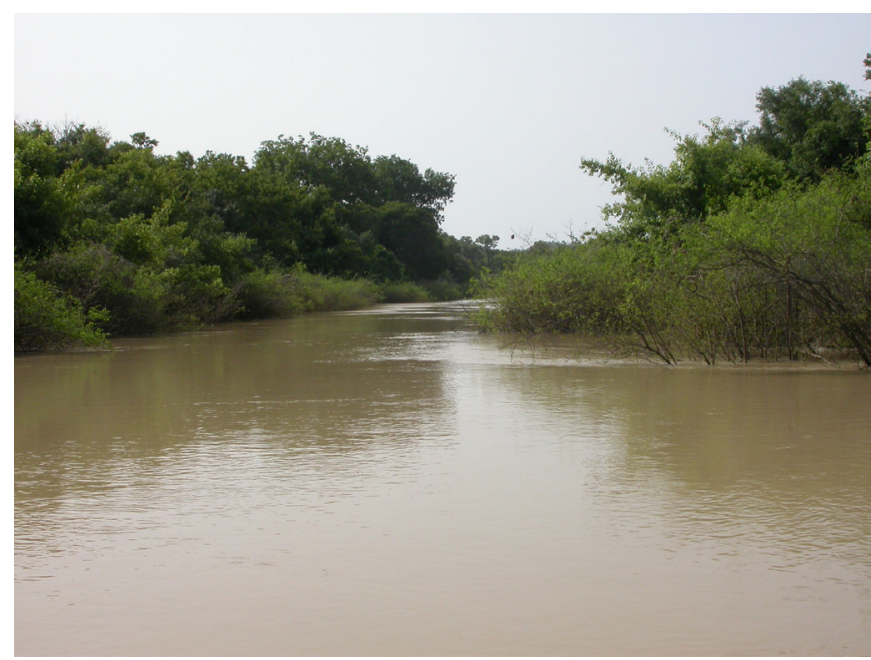

FIGURE 2. Gallery forest along the Arly river with Ziziphus spina-christi at the edge of the water.

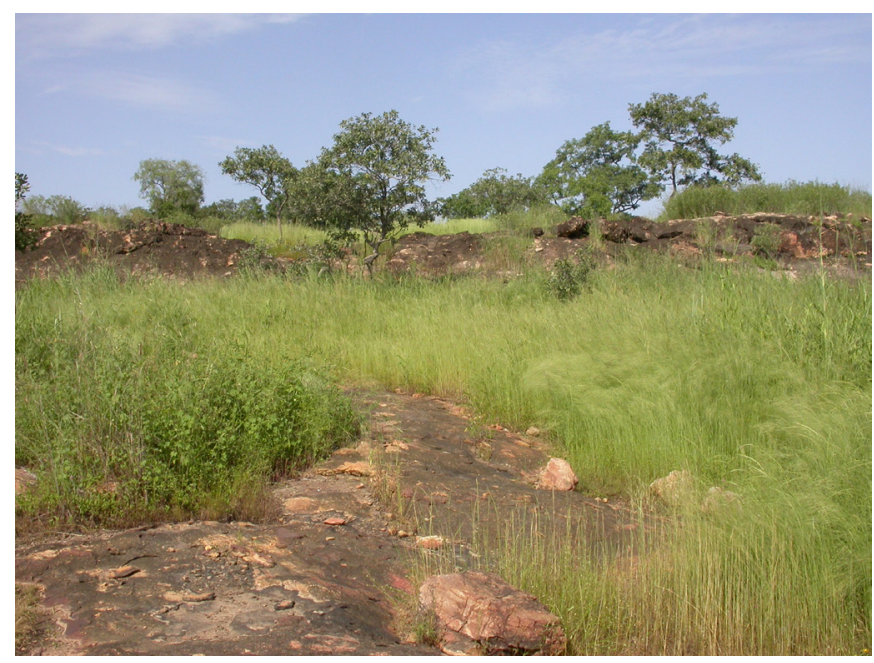

FIGURE 3. Sandstone hills of the Gobnangou mountains near the eastern entrance to the Arly NP. 
neighbouring areas of the so-called WAP complex - the Pama reserve (Mbayngone et al. 2008) and the Niger part of the $W$ transboundary park (Arbonnier et al. 2002), another sudanian site in the SW of Burkina Faso - the classified forest of Kou (Guinko and Thiombiano 2005) and the country's largest protected area - the Sahel reserve (Schmidt et al. 2008).

Most similar are Pama and Arly, grouping together with the W Park. As the geographic position in the center might suggest, Arly is more similar to either Pama or W Park, than these are to each other and has the fewest species exclusively found within its area. The mentioned three areas of the WAP complex are most similar to the Kou forest, the closer they are geographically. The environmental differences and larger distance to the other reserves makes the Sahel reserve the most distinctive in species composition. Although it has the lowest total species richness of the larger reserves (Kou forest is very small), it has the highest number of "exclusive" species.

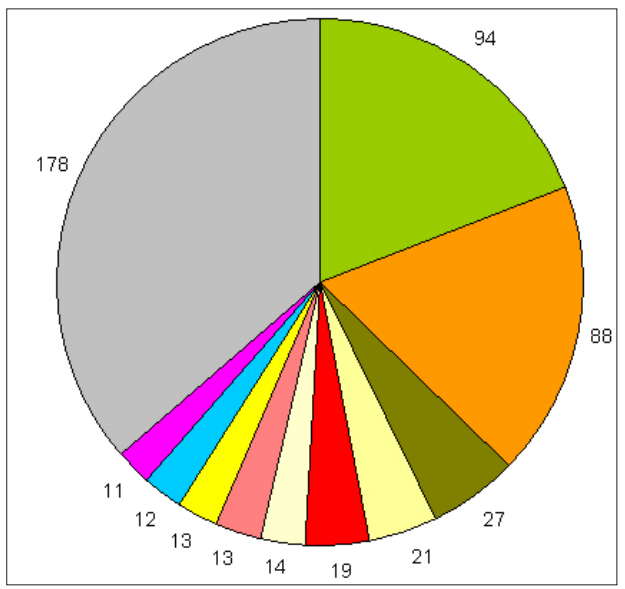

FIGURE 4. Family composition: Number of species within the ten largest families and the remaining 73 families. $\quad$ = Fabaceae; $\square=$ Poaceae; = Cyperaceae; = Rubiaceae $\square=$ Combretaceae; = Euphorbiaceae

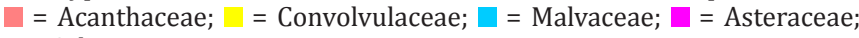
= Others.

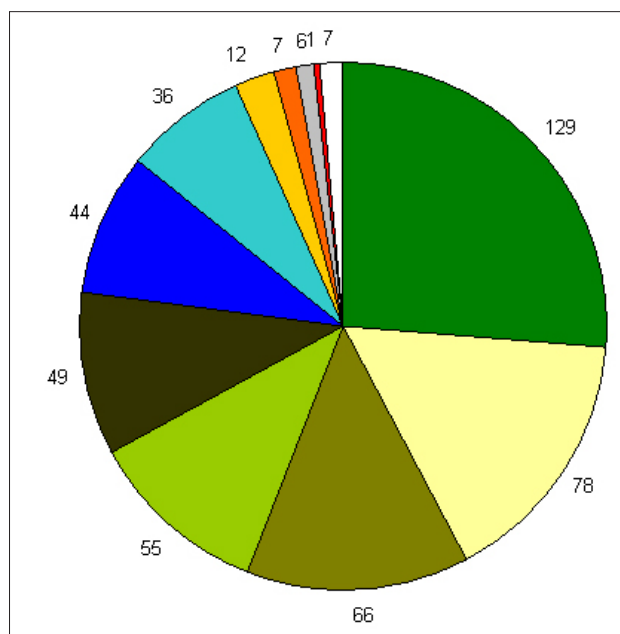

FIGURE 5. Life form composition: Number of species per life form.

$\square=$ Sudanian; $\square$ = Pantropical; $\square$ = Sudano-zambesian; $\square$ = Paleotropical; $\mathbf{\square}=$ Afrotropical; $\boldsymbol{\square}=$ Pluriregional African; $\square=$ Sudano-Guinean and Guinean-Congolian; $\square=$ Afro-American; $\square=$ Afro-Malagasy; $\square$ = Cultivated; $\square=$ Cosmopolitan; $\square=$ Unknown.

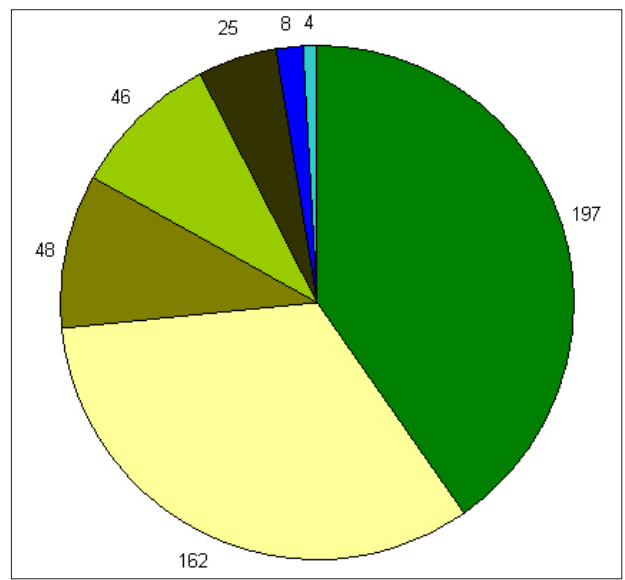

FIGURE 6. Chorological spectrum of the flora.

$\square=$ phanerophyte; $\square=$ therophyte; $\square=$ chamaephyte; $\square=$ hemicryptophyte; = geophyte; $\boldsymbol{\square}=$ hydrophyte; $\square=$ helophyte.

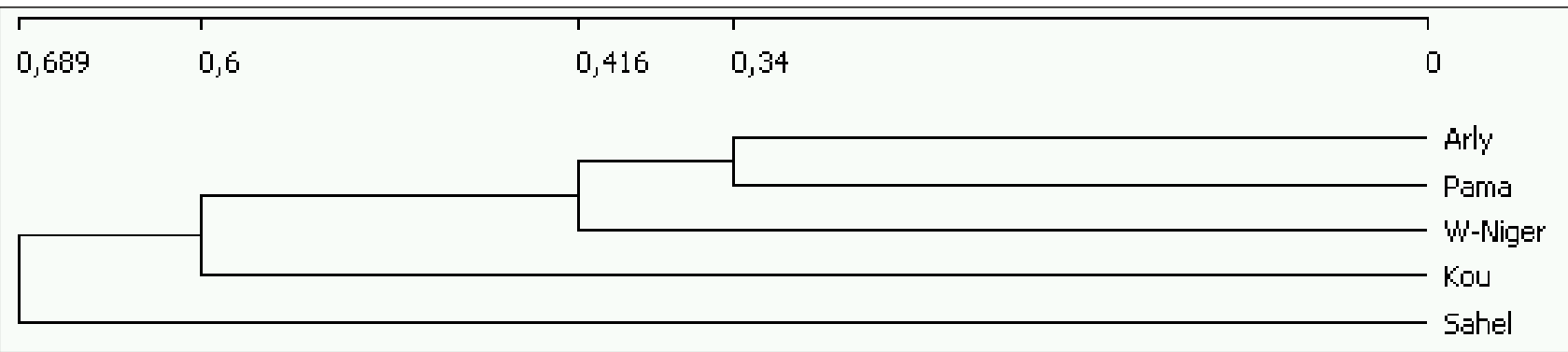

FIGURE 7. Dendrogram of the protected areas (average linkage, 1 - Sørensen, calculated with Community Analysis Package).

TABLE 1. Comparison of the protected areas: Sørensen index, species in common between two areas, species only found in the respective area, total species richness. Due to the use of a consistent synonymy, species richness might differ slightly from the original publications.

\begin{tabular}{|c|c|c|c|c|c|c|c|c|c|c|}
\hline & \multicolumn{4}{|c|}{ SøRENSEN INDEX } & \multicolumn{4}{|c|}{ SPECIES IN COMMON } & \multirow{2}{*}{$\begin{array}{l}\text { EXCLUSIVE } \\
\text { SPECIES }\end{array}$} & \multirow{2}{*}{$\begin{array}{l}\text { SPECIES } \\
\text { RICHNESS }\end{array}$} \\
\hline & Arly & Kou & Pama & Sahel & Arly & Kou & Pama & Sahel & & \\
\hline Arly & & & & & & & & & 69 & 490 \\
\hline Kou & 0,42 & & & & 162 & & & & 81 & 276 \\
\hline Pama & 0,66 & 0,43 & & & 311 & 156 & & & 72 & 450 \\
\hline Sahel & 0,36 & 0,14 & 0,33 & & 150 & 43 & 132 & & 130 & 347 \\
\hline W-Niger & 0,62 & 0,35 & 0,55 & 0,42 & 310 & 137 & 263 & 178 & 116 & 509 \\
\hline
\end{tabular}


TABLE 2. Magnoliophyta of the Arly National Park, ordered by family and species. Life form, chorological information and voucher specimens are provided for each species (life forms: [c] chamaephyte, [g] geophyte, [hl] helophyte, [hc] hemicryptophyte, [hy] hydrophyte, [p] phanerophyte, [t] therophyte; chorology: [S] sudanian, [Pan] pantropical, [SZ] sudano-zambesian, [Paleo] paleotropical, [TA] afrotropical, [PRA] pluriregional African, [SG-GC] sudano-guinean and guineo-congolian, [AA] afro-american, [AM] afro-malagasy, [Cu] cultivated, [Cosm] cosmopolitan).

\begin{tabular}{|c|c|c|c|}
\hline FAMILY AND SPECIES & $\mathbf{L F}$ & CHOR. & VOUCHER SPECIMENS \\
\hline \multicolumn{4}{|l|}{ ACANTHACEAE } \\
\hline Blepharis linariifolia Pers. & $\mathrm{t}$ & SZ & O. Ouédraogo 266 \\
\hline Blepharis maderaspatensis (L.) Heyne ex Roth & $\mathrm{p}$ & Pan & O. Ouédraogo 198 \\
\hline Dicliptera paniculata (Forssk.) I.Darbysh. & $\mathrm{t}$ & PRA & \\
\hline Dyschoriste heudelotiana (Nees) Kuntze & $\mathrm{c}$ & $\mathrm{TA}$ & O. Ouédraogo 217 \\
\hline Dyschoriste nagchana (Nees) Bennet & $\mathrm{c}$ & Pan & O. Ouédraogo 87, Küppers 1145 \\
\hline Hygrophila auriculata (Schumach.) Heine & $\mathrm{t}$ & Pan & \\
\hline Hygrophila senegalensis T.Anderson & $\mathrm{t}$ & SZ & O. Ouédraogo $142,143,147,226,320$ \\
\hline Justicia flava (Forssk.) Vahl & $\mathrm{p}$ & SZ & 0. Ouédraogo 273 \\
\hline Justicia ladanoides Lam. & $\mathrm{t}$ & $\mathrm{TA}$ & O. Ouédraogo 334 \\
\hline Lepidagathis anobrya Nees & $\mathrm{p}$ & $S$ & 0. Ouédraogo 152, 159 \\
\hline Lepidagathis collina (Endl.) Milne-Redh. & $\mathrm{p}$ & S & 0. Ouédraogo 312 \\
\hline Monechma ciliatum (Jacq.) Milne-Redh. & $\mathrm{c}$ & $\mathrm{TA}$ & \\
\hline Nelsonia canescens (Lam.) Spreng. & $\mathrm{c}$ & Pan & 0. Ouédraogo 84, 85, 168 \\
\hline
\end{tabular}

\section{Alismataceae}

Sagittaria guayanensis Kunth

hy Pan

\section{AMARANTHACEAE}

Achyranthes aspera L.

Alternanthera nodiflora $\mathrm{R}$. Br.

Alternanthera sessilis (L.) R.Br. ex DC.

Celosia trigyna L.

Pandiaka angustifolia (Vahl) Hepper

Pupalia lappacea (L.) Juss.

$\begin{array}{cc}\mathrm{t} & \text { Pan } \\ \mathrm{t} & \mathrm{SZ} \\ \mathrm{c} & \text { Pan } \\ \mathrm{t} & \text { PRA } \\ \mathrm{t} & \mathrm{SZ} \\ \mathrm{p} & \text { Pan }\end{array}$

O. Ouédraogo 201

0. Ouédraogo 166

O. Ouédraogo 98, 99, 100, 172

0. Ouédraogo 289

O. Ouédraogo $54,55,247$

\section{ANACARDIACEAE}

Lannea acida A. Rich.

Lannea barteri (Oliv.) Engl.

Lannea microcarpa Engl. \& K. Krause

Ozoroa insignis Delile

Sclerocarya birrea (A. Rich.) Hochst.

$\begin{array}{cc}\mathrm{p} & \mathrm{S} \\ \mathrm{p} & \mathrm{S} \\ \mathrm{p} & \mathrm{SZ} \\ \mathrm{p} & \mathrm{S} \\ \mathrm{p} & \mathrm{S}\end{array}$

\section{ANNONACEAE}

Annona senegalensis Pers.

Uvaria chamae P. Beauv

Xylopia parviflora (A.Rich.) Benth.

\section{ANTHERICACEAE}

Chlorophytum limosum (Baker) Nordal

\section{APOCYNACEAE}

Baissea multiflora A. DC.

Strophanthus sarmentosus DC.

\section{ARACEAE}

Culcasia saxatilis A.Chev.

Stylochaeton hypogaeus Lepr.

Stylochaeton lancifolius Kotschy \& Peyr.

\section{ARECACEAE}

Borassus aethiopum Mart.

\section{ASCLEPIADACEAE}

Brachystelma exile Bullock

Caralluma adscendens (Roxb.) Haw.

Cryptolepis oblongifolia (Meisn.) Schltr.

$\begin{array}{ccl}\mathrm{p} & \mathrm{S} & \text { Küppers 1147,1155 } \\ \mathrm{p} & \text { PRA } & \text { O. Ouédraogo 110, 111 } \\ \mathrm{p} & \text { GC } & \text { Schmidt } \text { et al. } 6244\end{array}$

Küppers 1135

g ? $\quad$ o. Ouédraogo 26,27

$\begin{array}{lll}\mathrm{p} & \text { SZ } & \text { Küppers } 1152, \text { Schmidt et al. } 6250 \\ \mathrm{p} & \text { GC } & \text { Schmidt } 843\end{array}$

$\begin{array}{ccc}\mathrm{p} & \mathrm{SG} & \text { O. Ouédraogo 330, } 351 \\ \mathrm{~g} & \mathrm{~S} & \\ \mathrm{~g} & \mathrm{~S} & \end{array}$

p $\quad$ SZ

$\begin{array}{ccc}\text { g } & \text { S } & \text { O. Ouédraogo } 495 \\ \text { c } & \text { SZ } & \\ \text { p } & \text { PRA } & \text { O. Ouédraogo } 391\end{array}$


TABLE 2. Continued.

\begin{tabular}{|c|c|c|c|}
\hline FAMILY AND SPECIES & LF & CHOR. & VOUCHER SPECIMENS \\
\hline Leptadenia hastata (Pers.) Decne. & $\mathrm{p}$ & PRA & 0. Ouédraogo 31, 32, 33 \\
\hline Oxystelma bornouense R.Br. & $\mathrm{p}$ & $\mathrm{TA}$ & O. Ouédraogo 300 \\
\hline \multicolumn{4}{|l|}{ Raphionacme brownii Scott-Elliot g, S] } \\
\hline Tacazzea apiculata Oliv. & $\mathrm{p}$ & $\mathrm{TA}$ & O. Ouédraogo 454 \\
\hline
\end{tabular}

\section{ASPARAgACEAE}

Asparagus africanus Lam.

g $\quad \mathrm{Pal}$

\section{ASTERACEAE}

Acanthospermum hispidum DC.

Aspilia bussei O.Hoffm. \& Muschl.

Aspilia paludosa Berhaut

Bidens borianiana (Sch.Bip. ex Schweinf. \& Asch.) Cufod.

Bidens engleri O.E.Schulz

Pan

Bidens ternata (Chiov.)Sherff

Pseudoconyza viscosa (Mill.) D’Arcy

Herderia truncata Cass.

Pentanema indicum (L.) Y.Ling

Tridax procumbens $\mathrm{L}$.

Vernonia nigritiana Oliv. \& Hiern

\section{BigNONIACEAE}

Stereospermum kunthianum Cham

\section{BOMBACACEAE}

Adansonia digitata L.

Bombax costatum Pellegr. \& Vuillet

Ceiba pentandra (L.) Gaertn.

\section{BoraginaCEAE}

Coldenia procumbens L.

Heliotropium indicum L.

Heliotropium strigosum Willd.

\section{BURSERACEAE}

Boswellia dalzielii Hutch.

Commiphora africana (A.Rich.) Engl.

BuTOMACEAE

Butomopsis latifolia (D.Don) Kunth

CAMpanulaceae

Sphenoclea zeylanica Gaertn.

\section{CAPPARIDACEAE}

Cadaba farinosa Forssk.

Capparis sepiaria L.

Capparis tomentosa Lam.

Cleome viscosa $\mathrm{L}$.

Crateva adansonii DC.

Maerua angolensis DC.

\section{CARYOPHYLlaceaE}

Polycarpaea corymbosa (L.) Lam.

Polycarpaea eriantha Hochst. ex A.Rich.

Polycarpaea linearifolia (DC.) DC.

\section{Celastraceae}

Gymnosporia senegalensis (Lam.) Loes.

Loeseneriella africana (Willd.) N.Hallé

Salacia owabiensis Hoyle

al

an

$\mathrm{t} \quad \mathrm{S}$

$\mathrm{t} \quad \mathrm{S}$

$\mathrm{t} \quad \mathrm{S}$

O. Ouédraogo 168, 225

O. Ouédraogo 200

O. Ouédraogo 203

$\mathrm{t} \quad \mathrm{s}$

t SG

$\mathrm{t} \quad \mathrm{SG}$

$\mathrm{t} \quad \mathrm{TA}$

c Pan

hc SZ O. Ouédraogo 20,21, 22, 23, 24

O. Ouédraogo 134

$\mathrm{p} \quad \mathrm{SZ}$

p $\quad S$

$\begin{array}{cc}\mathrm{p} & \mathrm{S} \\ \mathrm{p} & \mathrm{S} \\ \mathrm{p} & \mathrm{Pan}\end{array}$

p Pan

$\begin{array}{ccl}\text { t } & \text { SZ } & \text { O. Ouédraogo 137, 138, 139 } \\ \text { t } & \text { Pan } & \text { O. Ouédraogo 90, 91, 92 } \\ \text { c } & \text { Paleo } & \text { O. Ouédraogo } 76,165,170,174,367\end{array}$

$\begin{array}{cc}\mathrm{p} & \mathrm{S} \\ \mathrm{p} & \mathrm{Pal}\end{array}$

hl Paleo O. Ouédraogo 335

t Pan

$\begin{array}{cc}\mathrm{p} & \text { Paleo } \\ \mathrm{p} & \mathrm{SZ} \\ \mathrm{p} & \mathrm{S} \\ \mathrm{t} & \mathrm{SG} \\ \mathrm{p} & \text { Pal } \\ \mathrm{p} & \mathrm{TA}\end{array}$

Küppers 705

$\begin{array}{lll}\mathrm{t} & \text { Pal } & \\ \mathrm{t} & \mathrm{SZ} & \\ \mathrm{t} & \mathrm{TA} & \text { O. Ouédraogo 221, 244, } 328\end{array}$

$\begin{array}{lll}\mathrm{p} & \mathrm{SZ} & \\ \mathrm{p} & \mathrm{TA} & \text { O. Ouédraogo } 67,68,69 \\ \mathrm{p} & \text { GC } & \text { A. Ouédraogo } 315\end{array}$

Check List | Volume 7 | Issue 1 | 2011 
TABle 2. Continued.

\begin{tabular}{|c|c|c|c|}
\hline FAMILY AND SPECIES & $\mathbf{L F}$ & CHOR. & VOUCHER SPECIMENS \\
\hline \multicolumn{4}{|l|}{ Chrysobalanaceae } \\
\hline Parinari congensis Didr. & $\mathrm{p}$ & GC & O. Ouédraogo 118, 119, 120, Thiombiano et al. 3287 \\
\hline Parinari curatellifolia Planch. ex Benth. & $\mathrm{p}$ & SZ & \\
\hline \multicolumn{4}{|l|}{ COCHLOSPERMACEAE } \\
\hline Cochlospermum planchonii Hook. f. & $\mathrm{p}$ & S & \\
\hline Cochlospermum tinctorium A.Rich. & $\mathrm{p}$ & S & Thiombiano et al. 3282 \\
\hline \multicolumn{4}{|l|}{ COMBRETACEAE } \\
\hline Anogeissus leiocarpa (DC.) Guill. \& Perr. & $\mathrm{p}$ & SZ & Schmidt et al. 6255, 6256, 6269 \\
\hline Combretum aculeatum Vent. & $\mathrm{p}$ & SZ & \\
\hline Combretum acutum M.A.Lawson & $\mathrm{p}$ & S & O. Ouédraogo 95, 96, 97 \\
\hline Combretum adenogonium Steud. ex A. Rich. & $\mathrm{p}$ & S & Thiombiano et al. 2047 \\
\hline Combretum collinum Fresen. & $\mathrm{p}$ & $\mathrm{TA}$ & \\
\hline Combretum glutinosum Perr. ex DC. & $\mathrm{p}$ & S & \\
\hline Combretum micranthum G.Don & $\mathrm{p}$ & S & \\
\hline Combretum molle R.Br. ex G.Don & $\mathrm{p}$ & $\mathrm{TA}$ & Thiombiano et al. 2011 \\
\hline Combretum nigricans Lepr. ex Guill. \& Perr. & $\mathrm{p}$ & S & \\
\hline Combretum nioroense Aubrév. ex Keay & $\mathrm{p}$ & $S$ & O. Ouédraogo 503, Küppers 1148, Thiombiano 2009 \\
\hline Combretum paniculatum Vent. & $\mathrm{p}$ & PRA & \\
\hline Combretum sericeum G.Don & hc & S & O. Ouédraogo 108, 109 \\
\hline Guiera senegalensis J.F.Gmel. & $\mathrm{p}$ & SZ & \\
\hline Pteleopsis suberosa Engl. \& Diels & $\mathrm{p}$ & PRA & Thiombiano et al. 3284 \\
\hline Terminalia avicennioides Guill. \& Perr. & $\mathrm{p}$ & S & O. Ouédraogo 163, 186 \\
\hline Terminalia laxiflora Engl. & $\mathrm{p}$ & S & 0. Ouédraogo 502 \\
\hline Terminalia macroptera Guill. \& Perr. & $\mathrm{p}$ & S & \\
\hline Terminalia mollis M.A.Lawson & $\mathrm{p}$ & PRA & A. Ouédraogo 33 \\
\hline Terminalia schimperiana Hochst. & $\mathrm{p}$ & SG & \\
\hline \multicolumn{4}{|l|}{ COMMELINACEAE } \\
\hline Aneilema paludosum A.Chev. & hl & SZ & 0. Ouédraogo 270, 347 \\
\hline Commelina benghalensis $\mathrm{L}$. & c & Pal & \\
\hline Commelina erecta $\mathrm{L}$. & $\mathrm{t}$ & Pan & \\
\hline Commelina nigritana Benth. & $\mathrm{t}$ & $\mathrm{TA}$ & 0. Ouédraogo 314 \\
\hline Cyanotis longifolia Benth. & hc & SG & \\
\hline Murdannia simplex (Vahl) Brenan & $\mathrm{p}$ & Pal & O. Ouédraogo 311 \\
\hline \multicolumn{4}{|l|}{ CONVALLARIACEAE } \\
\hline Eriospermum flagelliforme (Baker) J.C.Manning & g & $\mathrm{TA}$ & O. Ouédraogo 180, 181 \\
\hline \multicolumn{4}{|l|}{ Convolvulaceae } \\
\hline Evolvulus alsinoides (L.) L. & c & Pan & \\
\hline Ipomoea aquatica Forssk. & hy & Pan & O. Ouédraogo 188 \\
\hline Ipomoea argentaurata Hallier f. & $\mathrm{p}$ & S & O. Ouédraogo 183 \\
\hline Ipomoea coscinosperma Hochst. ex Choisy & $\mathrm{t}$ & SZ & \\
\hline Ipomoea eriocarpa R.Br. & $\mathrm{t}$ & Pal & Küppers 1146 \\
\hline Ipomoea heterotricha Didr. & $\mathrm{t}$ & SZ & \\
\hline Ipomoea involucrata P.Beauv. & $\mathrm{t}$ & $\mathrm{TA}$ & O. Ouédraogo 460 \\
\hline Ipomoea marginata (Desr.) Verdc. & $\mathrm{p}$ & $?$ & O. Ouédraogo 280, 283, 285 \\
\hline Ipomoea vagans Baker & $\mathrm{t}$ & S & 0. Ouédraogo 237 \\
\hline Ipomoea verticillata Forsk. & $\mathrm{p}$ & S & 0. Ouédraogo 303 \\
\hline Merremia hederacea (Burm. f.) Hallier f. & $\mathrm{p}$ & Pan & O. Ouédraogo 164, 366, Schmidt et al. 6246 \\
\hline Merremia kentrocaulos (C.B.Clarke) Hallier f. & $\mathrm{p}$ & Pal & 0. Ouédraogo 470 \\
\hline Merremia pinnata (Hochst. ex Choisy) Hallier f. & $\mathrm{t}$ & PRA & 0. Ouédraogo 256, Schmidt et al. 6235 \\
\hline
\end{tabular}


TABle 2. Continued.

\begin{tabular}{|c|c|c|c|}
\hline FAMILY AND SPECIES & LF & CHOR. & VOUCHER SPECIMENS \\
\hline Kalanchoe laciniata (L.) DC. & $\mathrm{t}$ & Pan & O. Ouédraogo 499 \\
\hline \multicolumn{4}{|l|}{ CUCURBItACEAE } \\
\hline Cucumis maderaspatanus $\mathrm{L}$. & hc & Pal & \\
\hline Luffa cylindrica (L.) M.J.Roem. & $\mathrm{t}$ & AA & O. Ouédraogo 370, Schmidt 844 \\
\hline \multicolumn{4}{|l|}{ CYPERACEAE } \\
\hline Abildgaardia abortiva (Steud.) Lye & hc & PRA & \\
\hline Abildgaardia coleotricha (Hochst. ex A.Rich.) Lye & $\mathrm{t}$ & SG & \\
\hline Abildgaardia filamentosa (Vahl) Lye & hc & $\mathrm{S}$ & Schmidt et al. 6236 \\
\hline Abildgaardia hispidula (Vahl) Lye & hc & PRA & \\
\hline Cyperus cuspidatus Kunth & $\mathrm{t}$ & Pan & O. Ouédraogo 275 \\
\hline Cyperus difformis L. & $\mathrm{t}$ & Pan & O. Ouédraogo 305, 340 \\
\hline Cyperus digitatus Roxb. & g & PRA & O. Ouédraogo 121, 122, 123, 175 \\
\hline Cyperus haspan L. & $\mathrm{t}$ & Pan & O. Ouédraogo 58, 59, 60 \\
\hline Cyperus imbricatus Retz. & hc & Pan & O. Ouédraogo 126, 127, 128 \\
\hline Cyperus iria L. & $\mathrm{t}$ & Pal & O. Ouédraogo 374 \\
\hline Cyperus maculatus Boeckeler & g & PRA & O. Ouédraogo 173, 176 \\
\hline Cyperus podocarpus Boeckeler & $\mathrm{t}$ & $\mathrm{S}$ & O. Ouédraogo 199, 202 \\
\hline Cyperus pustulatus Vahl & $\mathrm{t}$ & PRA & O. Ouédraogo 204 \\
\hline Cyperus reduncus Hochst. ex Boeckeler & $\mathrm{t}$ & Pan & O. Ouédraogo $8,9,10$ \\
\hline Cyperus rotundus L. & g & $\mathrm{TA}$ & \\
\hline Eleocharis acutangula (Roxb.) Schult. & hl & Pan & \\
\hline Fimbristylis dichotoma (L.) Vahl & hc & Pan & \\
\hline Fimbristylis ferruginea (L.) Vahl & hc & Pan & O. Ouédraogo 212 \\
\hline Kyllinga erecta Schumach. & g & $\mathrm{AM}$ & O. Ouédraogo 56 \\
\hline Lipocarpha chinensis (Osbeck) J.Kern & $\mathrm{t}$ & Pal & O. Ouédraogo 304 \\
\hline Lipocarpha filiformis (Vahl) Kunth & $\mathrm{t}$ & Pal & \\
\hline Lipocarpha kernii (Raymond) Goetgh. & $\mathrm{t}$ & $?$ & O. Ouédraogo 306 \\
\hline Mariscus cylindristachyus Steud. & hc & Pan & O. Ouédraogo 250, Küppers 1138 \\
\hline Pycreus macrostachyos (Lam.) J. Raynal & $\mathrm{t}$ & Pan & O. Ouédraogo 271, 323 \\
\hline Schoenoplectiella senegalensis (Hochst. ex Steud.) Lye & $\mathrm{t}$ & Pal & O. Ouédraogo 222, 254 \\
\hline Scleria pergracilis (Nees) Kunth & $\mathrm{t}$ & Pal & \\
\hline Scleria sphaerocarpa (E.A.Rob.) Napper & $\mathrm{t}$ & SZ & O. Ouédraogo 141 \\
\hline \multicolumn{4}{|l|}{ DIOSCOREACEAE } \\
\hline Dioscorea bulbifera L. & g & Pan & \\
\hline Dioscorea dumetorum (Kunth) Pax & g & SZ & \\
\hline Dioscorea minutiflora Engl. & g & GC & O. Ouédraogo 327 \\
\hline Dioscorea sagittifolia $\mathrm{Pax}$ & g & $\mathrm{S}$ & O. Ouédraogo 263 \\
\hline \multicolumn{4}{|l|}{ DRACAENACEAE } \\
\hline Sansevieria liberica Hort. ex Gérôme \& Labroy & $\mathrm{c}$ & $\mathrm{TA}$ & O. Ouédraogo 450 \\
\hline \multicolumn{4}{|l|}{ DROSERACEAE } \\
\hline Drosera indica L. & $\mathrm{t}$ & Pal & \\
\hline \multicolumn{4}{|l|}{ EBENACEAE } \\
\hline Diospyros mespiliformis Hochst. ex A..DC. & $\mathrm{p}$ & Pal & Schmidt et al. 6263,6268 \\
\hline \multicolumn{4}{|l|}{ EUPHORBIACEAE } \\
\hline Antidesma venosum E. Mey. ex Tul. & $\mathrm{p}$ & PRA & \\
\hline Bridelia ferruginea Benth. & $\mathrm{p}$ & SZ & \\
\hline Bridelia scleroneura Muell.Arg. & $\mathrm{p}$ & $\mathrm{S}$ & \\
\hline Caperonia serrata (Turcz.) C.Presl & $\mathrm{t}$ & $\mathrm{S}$ & \\
\hline Croton nigritanus Scott-Elliot & $\mathrm{p}$ & GC & O. Ouédraogo 190, 329 \\
\hline Euphorbia convolvuloides Hochst. ex Benth. & hc & $\mathrm{S}$ & \\
\hline
\end{tabular}


TABle 2. Continued.

\begin{tabular}{|c|c|c|c|}
\hline FAMILY AND SPECIES & LF & CHOR. & VOUCHER SPECIMENS \\
\hline Euphorbia sudanica A.Chev. & $\mathrm{p}$ & $\mathrm{S}$ & \\
\hline Euphorbia thymifolia L. & $\mathrm{c}$ & Pan & 0. Ouédraogo 31, 82, 83 \\
\hline Flueggea virosa (Roxb. ex Willd.) Voigt & $\mathrm{p}$ & Pal & \\
\hline Hymenocardia acida Tul. & $\mathrm{p}$ & $\mathrm{TA}$ & \\
\hline Phyllanthus amarus Schum. \& Thonn. & $\mathrm{p}$ & Pan & \\
\hline Phyllanthus reticulatus Poir. & $\mathrm{p}$ & $\mathrm{TA}$ & O. Ouédraogo 93, 94 \\
\hline Tragia laminularis Müll.Arg. & $\mathrm{p}$ & S & O. Ouédraogo 475 \\
\hline Tragia senegalensis Müll.Arg. & $\mathrm{p}$ & $\mathrm{S}$ & \\
\hline \multicolumn{4}{|l|}{ FABACEAE } \\
\hline Abrus melanospermus Hassk. & $\mathrm{p}$ & $\mathrm{TA}$ & O. Ouédraogo 135, 136, Küppers 1158 \\
\hline Acacia dudgeonii Craib ex Holland & $\mathrm{p}$ & S & \\
\hline Acacia erythrocalyx Brenan & $\mathrm{p}$ & TA & \\
\hline Acacia gerrardii Benth. & $\mathrm{p}$ & $\mathrm{S}$ & O. Ouédraogo 518, Neumann 668, Thiombiano 3289 \\
\hline Acacia gourmaensis A. Chev. & $\mathrm{p}$ & $\mathrm{S}$ & \\
\hline Acacia hockii De Wild. & $\mathrm{p}$ & PRA & \\
\hline Acacia macrostachya Rchb. ex DC. & $\mathrm{p}$ & $\mathrm{SZ}$ & \\
\hline Acacia seyal Delile & $\mathrm{p}$ & $\mathrm{S}$ & \\
\hline Acacia sieberiana DC. & $\mathrm{p}$ & TA & \\
\hline Aeschynomene crassicaulis Harms & $\mathrm{t}$ & $\mathrm{S}$ & O. Ouédraogo 372 \\
\hline Aeschynomene indica L. & $\mathrm{c}$ & Pal & O. Ouédraogo 469 \\
\hline Aeschynomene sensitiva Sw. & $\mathrm{c}$ & AA & \\
\hline Afzelia africana Sm. ex Pers. & $\mathrm{p}$ & $\mathrm{SZ}$ & O. Ouédraogo 34, Küppers 704 \\
\hline Albizia chevalieri Harms & $\mathrm{p}$ & $\mathrm{S}$ & \\
\hline Alysicarpus ovalifolius (Schum. \& Thonn.) J. Léonard & $\mathrm{c}$ & Pal & \\
\hline Alysicarpus rugosus (Willd.) DC. & $\mathrm{t}$ & $\mathrm{AM}$ & \\
\hline Burkea africana Hook. & $\mathrm{p}$ & $\mathrm{TA}$ & \\
\hline Cajanus kerstingii Harms & $\mathrm{p}$ & $\mathrm{S}$ & O. Ouédraogo 245 \\
\hline Cassia sieberiana DC. & $\mathrm{p}$ & $\mathrm{SZ}$ & Küppers 1136 \\
\hline Chamaecrista absus (L.) H.S.Irwin \& Barneby & $\mathrm{p}$ & Pal & \\
\hline Chamaecrista nigricans (Vahl) Greene & $\mathrm{p}$ & Pal & \\
\hline Chamaecrista pratensis (R.Vig.) Du Puy & $\mathrm{p}$ & Pal & \\
\hline Crotalaria atrorubens Hochst. ex Benth. & $\mathrm{c}$ & S & O. Ouédraogo 316 \\
\hline Crotalaria goreensis Guill. \& Perr. & $\mathrm{c}$ & $\mathrm{TA}$ & O. Ouédraogo 369 \\
\hline Crotalaria hyssopifolia Klotzsch & $\mathrm{t}$ & $\mathrm{S}$ & 0. Ouédraogo 332 \\
\hline Crotalaria leprieurii Guill. \& Perr. & $\mathrm{t}$ & $\mathrm{SZ}$ & O. Ouédraogo 224 \\
\hline Crotalaria macrocalyx Benth. & $\mathrm{t}$ & $\mathrm{S}$ & O. Ouédraogo $4,5,6,7$ \\
\hline Crotalaria microcarpa Hochst. ex Benth. & $\mathrm{c}$ & $\mathrm{S}$ & O. Ouédraogo 337, 350 \\
\hline Crotalaria naragutensis Hutch. & $\mathrm{c}$ & $\mathrm{S}$ & \\
\hline Crotalaria retusa L. & $\mathrm{t}$ & Pan & \\
\hline Daniellia oliveri (Rolfe) Hutch. \& Dalziel & $\mathrm{p}$ & SZ & Thiombiano et al. 3286 \\
\hline Desmodium gangeticum (L.) DC. & $\mathrm{c}$ & Pal & O. Ouédraogo 265 \\
\hline Desmodium hirtum Guill. \& Perr. & $\mathrm{t}$ & PRA & O. Ouédraogo 246 \\
\hline Desmodium ospriostreblum Chiov. & $\mathrm{t}$ & Cul & O. Ouédraogo 399 \\
\hline Desmodium setigerum (E.Mey.) Benth. ex Harv. & $\mathrm{c}$ & PRA & O. Ouédraogo 132, 133 \\
\hline Desmodium velutinum (Willd.) DC. & $\mathrm{c}$ & Pan & \\
\hline Detarium microcarpum Guill. \& Perr. & $\mathrm{p}$ & $\mathrm{S}$ & \\
\hline Dialium guineense Willd. & $\mathrm{p}$ & $\mathrm{GC}$ & O. Ouédraogo 519, Küppers 1151, 1154 \\
\hline Dichrostachys cinerea (L.) Wright \& Arn. & $\mathrm{p}$ & Pan & \\
\hline Entada africana Guill. \& Perr. & $\mathrm{p}$ & PRA & \\
\hline Eriosema glomeratum (Guill. \& Perr.) Hook & $\mathrm{p}$ & $\mathrm{TA}$ & O. Ouédraogo 390 \\
\hline
\end{tabular}


TABle 2. Continued.

\begin{tabular}{|c|c|c|c|}
\hline FAMILY AND SPECIES & LF & CHOR. & VOUCHER SPECIMENS \\
\hline Eriosema griseum Baker & c & GC & O. Ouédraogo 310 \\
\hline Eriosema psoraleoides (Lam.) G. Don & $\mathrm{C}$ & PRA & O. Ouédraogo 361 \\
\hline Erythrina senegalensis A.DC. & $\mathrm{p}$ & GC & \\
\hline Indigofera bracteolata DC. & $\mathrm{p}$ & S & O. Ouédraogo 179, 261, 309 \\
\hline Indigofera colutea (Burm.f.) Merr. & $\mathrm{t}$ & Pal & \\
\hline Indigofera congesta Welw. ex Baker & $\mathrm{t}$ & PRA & O. Ouédraogo 86 \\
\hline Indigofera congolensis De Wild. \& T. Durand & $\mathrm{t}$ & $\mathrm{TA}$ & O. Ouédraogo 415 \\
\hline Indigofera dendroides Jacq. & $\mathrm{t}$ & $\mathrm{TA}$ & \\
\hline Indigofera geminata Baker & $\mathrm{t}$ & S & \\
\hline Indigofera hirsuta $\mathrm{L}$. & $\mathrm{t}$ & Pan & O. Ouédraogo 288 \\
\hline Indigofera leprieurii Baker f. & $\mathrm{t}$ & $\mathrm{SZ}$ & \\
\hline Indigofera macrocalyx Guill. \& Perr. & c & S & O. Ouédraogo 36, 37 \\
\hline Indigofera microcarpa Desv. & $\mathrm{t}$ & $\mathrm{AM}$ & O. Ouédraogo 124, 125 \\
\hline Indigofera omissa J.B.Gillett & $\mathrm{p}$ & $\mathrm{S}$ & \\
\hline Indigofera paniculata Vahl ex Pers. & $\mathrm{t}$ & $\mathrm{TA}$ & O. Ouédraogo 15, 290, 296, 302, Küppers 1159 \\
\hline Indigofera pilosa Poir. & $\mathrm{t}$ & $\mathrm{SZ}$ & O. Ouédraogo 11 \\
\hline Indigofera secundiflora Poir. & $\mathrm{t}$ & Pal & O. Ouédraogo 362 \\
\hline Indigofera senegalensis Lam. & $\mathrm{t}$ & $\mathrm{S}$ & O. Ouédraogo 197 \\
\hline Indigofera stenophylla Guill. \& Perr. & $\mathrm{t}$ & $\mathrm{S}$ & \\
\hline Indigofera tinctoria $\mathrm{L}$. & $\mathrm{p}$ & PRA & \\
\hline Indigofera trichopoda Lepr. ex Guill. \& Perr. & $\mathrm{t}$ & $\mathrm{S}$ & Schmidt et al. 6267 \\
\hline Isoberlinia doka Craib \& Stapf & $\mathrm{p}$ & S & \\
\hline Mimosa pigra $\mathrm{L}$. & $\mathrm{p}$ & Pan & Küppers 1153 \\
\hline Parkia biglobosa (Jacq.) Benth. & $\mathrm{p}$ & Pal & \\
\hline Pericopsis laxiflora (Benth.) Meeuwen & $\mathrm{p}$ & $\mathrm{S}$ & \\
\hline Philenoptera laxiflora (Guill. \& Perr.) Roberty & $\mathrm{p}$ & PRA & A. Ouédraogo 300, Thiombiano et al. 3290 \\
\hline Piliostigma reticulatum (DC.) Hochst. & $\mathrm{p}$ & $\mathrm{S}$ & \\
\hline Piliostigma thonningii (Schumach.) Milne-Redh. & $\mathrm{p}$ & TA & \\
\hline Prosopis africana (Guill. \& Perr.) Taub. & $\mathrm{p}$ & $\mathrm{S}$ & \\
\hline Pterocarpus erinaceus Poir. & $\mathrm{p}$ & SZ & A. Ouédraogo 299 \\
\hline Pterocarpus santalinoides L’Hér. ex DC. & $\mathrm{p}$ & PRA & O. Ouédraogo 177, 192, A. Ouédraogo 31 \\
\hline Rhynchosia minima (L.) DC. & $\mathrm{p}$ & Pan & O. Ouédraogo 241 \\
\hline Rhynchosia sublobata (Schumach. \& Thonn.) Meikle & $\mathrm{p}$ & PRA & \\
\hline Senna obtusifolia (L.) H.S.Irwin \& Barneby & $\mathrm{p}$ & Pan & \\
\hline Sesbania rostrata Bremek. \& Oberm. & $\mathrm{t}$ & $\mathrm{S}$ & \\
\hline Sesbania sesban (L.) Merr. & $\mathrm{p}$ & Pal & \\
\hline Stylosanthes erecta P.Beauv. & c & Gc & \\
\hline Tamarindus indica L. & $\mathrm{p}$ & Pan & \\
\hline Tephrosia bracteolata Guill. \& Perr. & $\mathrm{p}$ & SZ & O. Ouédraogo 151 \\
\hline Tephrosia elegans Schumach. & $\mathrm{p}$ & SZ & O. Ouédraogo 307 \\
\hline Tephrosia linearis (Willd.) Pers. & $\mathrm{t}$ & $\mathrm{AM}$ & O. Ouédraogo $34,38,286$ \\
\hline Tephrosia mossiensis A.Chev. & $\mathrm{p}$ & $\mathrm{S}$ & O. Ouédraogo 232 \\
\hline Tephrosia nana Kotschy ex Schweinf. & $\mathrm{p}$ & $\mathrm{GC}$ & O. Ouédraogo 233 \\
\hline Tephrosia pedicellata Baker & c & $\mathrm{S}$ & \\
\hline Uraria picta (Jacq.) DC. & $\mathrm{p}$ & Pal & \\
\hline Vigna filicaulis Hepper & $\mathrm{p}$ & SZ & O. Ouédraogo 140, 155, 214, 336 \\
\hline Vigna heterophylla A.Rich. & $\mathrm{p}$ & $\mathrm{SZ}$ & O. Ouédraogo 456 \\
\hline Vigna longifolia (Benth.) Verdc. & $\mathrm{p}$ & GC & \\
\hline Vigna multinervis Hutch. \& Dalziel & $\mathrm{p}$ & $\mathrm{S}$ & O. Ouédraogo 463 \\
\hline Vigna racemosa (G.Don) Hutch. \& Dalziel & $\mathrm{p}$ & GC & O. Ouédraogo 356 \\
\hline
\end{tabular}


TABle 2. Continued.

\begin{tabular}{|c|c|c|c|}
\hline FAMILY AND SPECIES & LF & CHOR. & VOUCHER SPECIMENS \\
\hline Vigna reticulata Hook. f. & $\mathrm{p}$ & PRA & O. Ouédraogo 472 \\
\hline Xeroderris stuhlmannii (Taub.) Mendonca \& E.C. Sousa & $\mathrm{p}$ & SZ & \\
\hline Zornia glochidiata Reichb. ex DC. & $\mathrm{t}$ & $\mathrm{TA}$ & \\
\hline \multicolumn{4}{|l|}{ FLACOURTIACEAE } \\
\hline Flacourtia indica (Burm.f.) Merr. & $\mathrm{p}$ & Cul & \\
\hline \multicolumn{4}{|l|}{ GUTTIFERAE } \\
\hline Garcinia livingstonei T.Anderson & $\mathrm{p}$ & SZ & \\
\hline Psorospermum glaberrimum Hochr. & $\mathrm{p}$ & $\mathrm{S}$ & O. Ouédraogo 520 \\
\hline \multicolumn{4}{|l|}{ HYACINTHACEAE } \\
\hline Albuca nigritana (Baker) Troupin & g & $\mathrm{S}$ & \\
\hline Dipcadi tacazzeanum (Hochst. ex A.Rich.) Baker & g & $\mathrm{S}$ & O. Ouédraogo 182 \\
\hline \multicolumn{4}{|l|}{ HYDROCHARITACEAE } \\
\hline Ottelia ulvifolia (Planch.) Walp. & hy & PRA & \\
\hline \multicolumn{4}{|l|}{ HYPOXIDACEAE } \\
\hline Curculigo pilosa (Schum. \& Thonn.) Engl. & g & PRA & \\
\hline \multicolumn{4}{|l|}{ LAMIACEAE } \\
\hline Englerastrum schweinfurthii Briq. & $\mathrm{t}$ & $\mathrm{S}$ & O. Ouédraogo 517 \\
\hline Hoslundia opposita Vahl & $\mathrm{p}$ & $\mathrm{AM}$ & \\
\hline Hyptis spicigera Lam. & $\mathrm{p}$ & Pan & \\
\hline Hyptis suaveolens Poit. & $\mathrm{c}$ & Pan & \\
\hline Leucas martinicensis (Jacq.) R.Br. & $\mathrm{t}$ & Pan & \\
\hline Ocimum obovatum E.Mey. ex Benth. & hc & SZ & O. Ouédraogo 349 \\
\hline Platostoma africanum P.Beauv. & $\mathrm{t}$ & Pal & \\
\hline Plectranthus gracillimus (T.C.E.Fr.) Hutch. \& Dandy & $\mathrm{t}$ & $\mathrm{S}$ & \\
\hline Tinnea barteri Gürke & $\mathrm{p}$ & SZ & \\
\hline \multicolumn{4}{|l|}{ LENTIBULARIACEAE } \\
\hline Utricularia stellaris L.f. & hy & $?$ & \\
\hline \multicolumn{4}{|l|}{ LILIACEAE } \\
\hline Drimia altissima (L.f.) Ker Gawl. & g & $\mathrm{S}$ & O. Ouédraogo 338 \\
\hline Scadoxus multiflorus (Martyn) Raf. & g & $\mathrm{TA}$ & O. Ouédraogo 105, 106, 107, 178 \\
\hline \multicolumn{4}{|l|}{ LOGANIACEAE } \\
\hline Strychnos innocua Delile & $\mathrm{p}$ & SZ & \\
\hline Strychnos spinosa Lam. & $\mathrm{p}$ & $\mathrm{AM}$ & \\
\hline \multicolumn{4}{|l|}{ LORANTHACEAE } \\
\hline Agelanthus dodoneifolius (DC.) Polhill \& Wiens & $\mathrm{p}$ & $\mathrm{S}$ & \\
\hline \multicolumn{4}{|l|}{ LYTHRACEAE } \\
\hline Ammannia auriculata Willd. & $\mathrm{t}$ & $\mathrm{S}$ & O. Ouédraogo 279 \\
\hline \multicolumn{4}{|l|}{ MALVACEAE } \\
\hline Cienfuegosia heteroclada Sprague & c & $\mathrm{S}$ & O. Ouédraogo 490 \\
\hline Hibiscus cannabinus L. & $\mathrm{t}$ & Cul & O. Ouédraogo 364 \\
\hline Hibiscus panduriformis Burm.f. & $\mathrm{t}$ & Pal & O. Ouédraogo 282 \\
\hline Hibiscus rostellatus Guill. \& Perr. & $\mathrm{t}$ & GC & O. Ouédraogo 363 \\
\hline Hibiscus scotellii Baker f. & $\mathrm{p}$ & GC & O. Ouédraogo 353 \\
\hline Sida acuta Burm. f. & c & Pan & \\
\hline Sida alba L. & $\mathrm{p}$ & Pan & \\
\hline Sida linifolia Juss. ex Cav. & $\mathrm{p}$ & $\mathrm{AA}$ & O. Ouédraogo 223 \\
\hline Sida rhombifolia L. & c & Pan & \\
\hline Sida urens L. & c & Pan & \\
\hline Urena lobata L. & c & Pan & O. Ouédraogo 195 \\
\hline Wissadula rostrata (Schumach.) Hook.f. & $\mathrm{t}$ & $\mathrm{AA}$ & \\
\hline
\end{tabular}


TABLE 2. Continued.

\begin{tabular}{|c|c|c|c|}
\hline FAMILY AND SPECIES & LF & CHOR. & VOUCHER SPECIMENS \\
\hline \multicolumn{4}{|l|}{ MARANTACEAE } \\
\hline Thalia geniculata L. & hl & PRA & O. Ouédraogo 269 \\
\hline \multicolumn{4}{|l|}{ MARSILEACEAE } \\
\hline Marsilea minuta L. & hy & ? & O. Ouédraogo 301 \\
\hline \multicolumn{4}{|l|}{ MELIACEAE } \\
\hline Azadirachta indica A. Juss. & $\mathrm{p}$ & Pal & \\
\hline Khaya senegalensis (Desr.) A. Juss. & $\mathrm{p}$ & $\mathrm{S}$ & \\
\hline Pseudocedrela kotschyi (Schweinf.) Harms & $\mathrm{p}$ & $\mathrm{S}$ & \\
\hline Trichilia emetica Vahl & $\mathrm{p}$ & SZ & \\
\hline \multicolumn{4}{|l|}{ MENISPERMACEAE } \\
\hline Cissampelos mucronata A. Rich. & $\mathrm{p}$ & SZ & O. Ouédraogo 114, 115, Thiombiano et al. 3288 \\
\hline Triclisia patens Oliv. & $\mathrm{p}$ & GC & Schmidt et al. 6252 \\
\hline Triclisia subcordata Oliv. & $\mathrm{p}$ & GC & Küppers 1144, 1157 \\
\hline \multicolumn{4}{|l|}{ MOLLUGINACEAE } \\
\hline Glinus lotoides L. & $\mathrm{t}$ & Pan & A. Ouédraogo 170 \\
\hline Mollugo nudicaulis Lam. & $\mathrm{t}$ & Pal & O. Ouédraogo 196, Schmidt et al. 6254 \\
\hline \multicolumn{4}{|l|}{ MORACEAE } \\
\hline Ficus abutilifolia (Miq.) Miq. & $\mathrm{p}$ & $\mathrm{S}$ & A. Ouédraogo 31 \\
\hline Ficus glumosa Delile & $\mathrm{p}$ & $\mathrm{S}$ & \\
\hline Ficus ingens (Miq.) Miq. & $\mathrm{p}$ & $\mathrm{SZ}$ & O. Ouédraogo 104, Küppers 1134, A. Ouédraogo 28 \\
\hline Ficus sur Forssk. & $\mathrm{p}$ & SG & \\
\hline Ficus sycomorus L. & $\mathrm{p}$ & $\mathrm{TA}$ & \\
\hline Ficus thonningii Blume & $\mathrm{p}$ & $\mathrm{TA}$ & \\
\hline \multicolumn{4}{|l|}{ MYRTACEAE } \\
\hline Syzygium guineense (Willd.) DC. & $\mathrm{p}$ & $\mathrm{TA}$ & O. Ouédraogo 346, 348, Küppers 1149, A. Ouédraogo 32 \\
\hline \multicolumn{4}{|l|}{ NYCTAGINACEAE } \\
\hline Boerhavia erecta $\mathrm{L}$. & $\mathrm{t}$ & Cosm & \\
\hline \multicolumn{4}{|l|}{ NYMPHAEACEAE } \\
\hline Nymphaea lotus L. & hy & Pan & \\
\hline Nymphaea micrantha Guill. \& Perr. & hy & GC & \\
\hline \multicolumn{4}{|l|}{ OLACACEAE } \\
\hline Ximenia americana L. & $\mathrm{p}$ & Pan & \\
\hline \multicolumn{4}{|l|}{ OLEACEAE } \\
\hline Jasminum obtusifolium Baker & $\mathrm{p}$ & S & \\
\hline \multicolumn{4}{|l|}{ ONAGRACEAE } \\
\hline Ludwigia abyssinica A.Rich. & $\mathrm{p}$ & $\mathrm{AM}$ & \\
\hline Ludwigia adscendens (L.) H.Hara & $\mathrm{c}$ & PRA & 0. Ouédraogo 88, 89 \\
\hline Ludwigia hyssopifolia (G. Don) Exell & $\mathrm{t}$ & S & O. Ouédraogo 459 \\
\hline Ludwigia octovalvis (Jacq.) P.H. Raven & c & PRA & 0. Ouédraogo 339 \\
\hline \multicolumn{4}{|l|}{ ORCHIDACEAE } \\
\hline Eulophia guineensis Lindl. & $\mathrm{g}$ & GC & \\
\hline \multicolumn{4}{|l|}{ OXALIDACEAE } \\
\hline Biophytum umbraculum Welw. & $\mathrm{t}$ & Pal & \\
\hline \multicolumn{4}{|l|}{ PEDAliaceae } \\
\hline Ceratotheca sesamoides Endl. & $\mathrm{t}$ & $\mathrm{SZ}$ & O. Ouédraogo 210 \\
\hline \multicolumn{4}{|l|}{ Plumbaginaceae } \\
\hline Plumbago zeylanica L. & $\mathrm{p}$ & Pan & O. Ouédraogo 344 \\
\hline \multicolumn{4}{|l|}{ PoAceae } \\
\hline Acroceras amplectens Stapf & $\mathrm{t}$ & $\mathrm{S}$ & \\
\hline Acroceras zizanioides (Kunth) Dandy & $\mathrm{c}$ & Pan & O. Ouédraogo 207 \\
\hline
\end{tabular}


TABle 2. Continued.

\begin{tabular}{|c|c|c|c|}
\hline FAMILY AND SPECIES & LF & CHOR. & VOUCHER SPECIMENS \\
\hline Anadelphia afzeliana (Rendle) Stapf & hc & $\mathrm{SZ}$ & O. Ouédraogo 432 \\
\hline Andropogon canaliculatus Schumach. & hc & $\mathrm{S}$ & \\
\hline Andropogon chinensis (Nees) Merr. & hc & Pal & O. Ouédraogo 25, 294, 297, 299 \\
\hline Andropogon fastigiatus Sw. & $\mathrm{t}$ & Pan & O. Ouédraogo 440 \\
\hline Andropogon gayanus Kunth & hc & $\mathrm{S}$ & O. Ouédraogo 272, 359 \\
\hline Andropogon pseudapricus Stapf & $\mathrm{t}$ & AA & 0. Ouédraogo 313, 317 \\
\hline Andropogon schirensis Hochst. ex A.Rich. & hc & PRA & \\
\hline Andropogon tectorum Schumach. \& Thonn. & hc & $\mathrm{S}$ & O. Ouédraogo 236, 239, 277, Schmidt et al. 6231 \\
\hline Aristida adscensionis L. & $\mathrm{t}$ & Pan & O. Ouédraogo $16,17,18,19$ \\
\hline Aristida kerstingii Pilg. & $\mathrm{t}$ & $\mathrm{S}$ & \\
\hline Brachiaria falcifera (Trin.) Stapf & hc & $\mathrm{S}$ & Küppers 1162 \\
\hline Brachiaria jubata (Fig. \& De Not.) Stapf & hc & SZ & \\
\hline Brachiaria lata (Schumach.) C.E.Hubb. & $\mathrm{t}$ & Pal & \\
\hline Brachiaria villosa (Lam.) A.Camus & $\mathrm{t}$ & $\mathrm{S}$ & O. Ouédraogo 185, 243, 259 \\
\hline Cenchrus biflorus Roxb. & $\mathrm{t}$ & $\mathrm{TA}$ & O. Ouédraogo 508 \\
\hline Chasmopodium caudatum (Hack.) Stapf & $\mathrm{t}$ & SZ & \\
\hline Chloris pilosa Schumach. & $\mathrm{t}$ & SZ & \\
\hline Chrysopogon nigritanus (Benth.) Veldkamp & hc & $\mathrm{SZ}$ & O. Ouédraogo 515 \\
\hline Ctenium newtonii Hack. & hc & $\mathrm{TA}$ & O. Ouédraogo 227 \\
\hline Cymbopogon caesius (Nees ex Hook. \& Arn.) Stapf & hc & $\mathrm{TA}$ & O. Ouédraogo 39, 278, 284 \\
\hline Cymbopogon schoenanthus (L.) Spreng. & hc & Pan & \\
\hline Dactyloctenium aegyptium (L.) Willd. & $\mathrm{t}$ & Pan & \\
\hline Digitaria ciliaris (Retz.) Koeler & $\mathrm{t}$ & Pan & 0. Ouédraogo 251, 252 \\
\hline Digitaria gayana (Kunth) Stapf ex A.Chev. & $\mathrm{t}$ & Pan & O. Ouédraogo 258 \\
\hline Diheteropogon amplectens (Nees) Clayton & hc & Pan & O. Ouédraogo 257 \\
\hline Echinochloa colona (L.) Link & $\mathrm{t}$ & Pan & \\
\hline Echinochloa stagnina (Retz.) P.Beauv. & $\mathrm{t}$ & PRA & O. Ouédraogo 331 \\
\hline Eleusine indica (L.) Gaertn. & $\mathrm{t}$ & Pan & \\
\hline Elionurus ciliaris Kunth & hc & $\mathrm{S}$ & O. Ouédraogo 417 \\
\hline Elytrophorus spicatus (Willd.) A.Camus & $\mathrm{t}$ & Pal & O. Ouédraogo 319, 341, A. Ouédraogo 65 \\
\hline Eragrostis ciliaris (L.) R.Br. & $\mathrm{t}$ & Pal & O. Ouédraogo $12,13,14$ \\
\hline Eragrostis egregia Clayton & hc & $\mathrm{S}$ & O. Ouédraogo 371 \\
\hline Eragrostis gangetica (Roxb.) Steud. & $\mathrm{t}$ & Pal & O. Ouédraogo 291, 333, 342 \\
\hline Eragrostis japonica (Thunb.) Trin. & $\mathrm{t}$ & TA & O. Ouédraogo 262 \\
\hline Eragrostis tenella (L.) P.Beauv. ex Roem. \& Schult. & $\mathrm{t}$ & Pal & \\
\hline Eragrostis tremula Hochst. ex Steud. & $\mathrm{t}$ & Pal & \\
\hline Eragrostis turgida (Schumach.) De Wild. & $\mathrm{t}$ & $\mathrm{S}$ & \\
\hline Euclasta condylotricha (Hochst. ex Steud.) Stapf & $\mathrm{t}$ & Pan & O. Ouédraogo 431 \\
\hline Hackelochloa granularis (L.) Kuntze & $\mathrm{t}$ & Pan & \\
\hline Heteropogon contortus (L.) P.Beauv. ex Roem. \& Schult. & hc & Pan & \\
\hline Heteropogon melanocarpus (Elliott) Benth. & $\mathrm{t}$ & Pan & O. Ouédraogo 325, Schmidt et al. 6243 \\
\hline Hyparrhenia barteri (Hack.) Stapf & $\mathrm{t}$ & $\mathrm{S}$ & Schmidt 838 \\
\hline Hyparrhenia cyanescens (Stapf) Stapf & hc & GC & O. Ouédraogo 154, 160, 161, 321 \\
\hline Hyparrhenia glabriuscula (Hochst. ex A.Rich.) Stapf & hc & SZ & O. Ouédraogo 381 \\
\hline Hyparrhenia involucrata Stapf & $\mathrm{t}$ & $\mathrm{S}$ & O. Ouédraogo 396 \\
\hline Hyparrhenia rufa (Nees) Stapf & $\mathrm{t}$ & Pan & O. Ouédraogo 57 \\
\hline Hyparrhenia smithiana (Hook.f.) Stapf & hc & $\mathrm{S}$ & \\
\hline Hyparrhenia subplumosa Stapf & hc & GC & Schmidt et al. 6239 \\
\hline Hyperthelia dissoluta (Nees ex Steud.) Clayton & hc & AA & \\
\hline Imperata cylindrica (L.) P.Beauv. & g & Pan & \\
\hline
\end{tabular}


TABLE 2. ContinUed.

\begin{tabular}{|c|c|c|c|}
\hline FAMILY AND SPECIES & LF & CHOR. & VOUCHER SPECIMENS \\
\hline Loudetia hordeiformis (Stapf) C.E.Hubb. & $\mathrm{t}$ & $\mathrm{S}$ & O. Ouédraogo 40, 41, 42, 235, Schmidt et al. 6242 \\
\hline Loudetia simplex (Nees) C.E. Hubb. & hc & SZ & \\
\hline Loudetia togoensis (Pilg.) C.E.Hubb. & $\mathrm{t}$ & $\mathrm{S}$ & O. Ouédraogo 393 \\
\hline Loudetiopsis kerstingii (Pilger) Conert & $\mathrm{t}$ & $\mathrm{S}$ & \\
\hline Microchloa indica (L. f.) P.Beauv. & $\mathrm{t}$ & Pan & \\
\hline Monocymbium ceresiiforme (Nees) Stapf & hc & $\mathrm{S}$ & O. Ouédraogo 308 \\
\hline Oryza barthii A.Chev. & $\mathrm{t}$ & $\mathrm{S}$ & O. Ouédraogo 194 \\
\hline Oryza glaberrima Steud. & $\mathrm{t}$ & Cul & O. Ouédraogo 267 \\
\hline Oxytenanthera abyssinica (A.Rich.) Munro & $\mathrm{p}$ & TA & \\
\hline Panicum anabaptistum Steud. & c & $\mathrm{S}$ & \\
\hline Panicum fluviicola Steud. & hc & PRA & O. Ouédraogo 358, Schmidt et al. 6238 \\
\hline Panicum maximum Jacq. & hc & Pan & O. Ouédraogo 255, 324 \\
\hline Panicum pansum Rendle & $\mathrm{t}$ & $\mathrm{SZ}$ & O. Ouédraogo 148, 149, 213, 238 \\
\hline Panicum phragmitoides Stapf & hc & $\mathrm{S}$ & 0. Ouédraogo $61,62,63$ \\
\hline Paspalum scrobiculatum L. & hc & Pal & O. Ouédraogo 293 \\
\hline Pennisetum pedicellatum Trin. & $\mathrm{t}$ & Pal & \\
\hline Pennisetum polystachion (L.) Schult. & $\mathrm{t}$ & Pan & \\
\hline Pennisetum unisetum (Nees) Benth. & hc & $\mathrm{TA}$ & 0. Ouédraogo $43,44,46$ \\
\hline Rhytachne triaristata (Steud.) Stapf & $\mathrm{t}$ & $\mathrm{SZ}$ & O. Ouédraogo 357 \\
\hline Rottboellia cochinchinensis (Lour.) Clayton & $\mathrm{t}$ & Pan & \\
\hline Sacciolepis africana C.E.Hubb. \& Snowden & g & GC & \\
\hline Schizachyrium brevifolium (Sw.) Nees ex Büse & $\mathrm{t}$ & Pan & \\
\hline Schizachyrium exile (Hochst.) Pilg. & $\mathrm{t}$ & $\mathrm{S}$ & \\
\hline Schizachyrium nodulosum (Hack.) Stapf & $\mathrm{t}$ & PRA & O. Ouédraogo 211 \\
\hline Schizachyrium sanguineum (Retz.) Alston & hc & Pan & O. Ouédraogo 153 \\
\hline Schoenefeldia gracilis Kunth & $\mathrm{t}$ & Pal & \\
\hline Setaria barbata (Lam.) Kunth & $\mathrm{t}$ & Pan & O. Ouédraogo 276 \\
\hline Setaria pumila (Poir.) Roem. \& Schult. & $\mathrm{t}$ & $\mathrm{TA}$ & \\
\hline Setaria sphacelata (Schumach.) Stapf \& C.E.Hubb. ex M.B.Moss & hc & TA & \\
\hline Sorghastrum bipennatum (Hack.) Pilg. & $\mathrm{t}$ & SZ & \\
\hline Sporobolus festivus Hochst. ex A.Rich. & hc & PRA & \\
\hline Sporobolus paniculatus (Trin.) T.Durand \& Schinz & $\mathrm{t}$ & $\mathrm{AA}$ & O. Ouédraogo 315 \\
\hline Sporobolus pectinellus Mez & $\mathrm{t}$ & $\mathrm{S}$ & \\
\hline Sporobolus pyramidalis P.Beauv. & hc & AA & O. Ouédraogo 511, Küppers 1160 \\
\hline Tripogon minimus (A.Rich.) Hochst. ex Steud. & $\mathrm{t}$ & PRA & O. Ouédraogo 408 \\
\hline Urelytrum annuum Stapf & $\mathrm{t}$ & $\mathrm{S}$ & O. Ouédraogo 414 \\
\hline \multicolumn{4}{|l|}{ Polygalaceae } \\
\hline Polygala arenaria Willd. & $\mathrm{t}$ & PRA & O. Ouédraogo 156 \\
\hline Polygala multiflora Poir. & $\mathrm{t}$ & $\mathrm{S}$ & O. Ouédraogo 189, Schmidt et al. 6233 \\
\hline Securidaca longipedunculata Fresen. & $\mathrm{p}$ & $\mathrm{TA}$ & \\
\hline
\end{tabular}

\begin{tabular}{|c|c|c|c|}
\hline \multicolumn{4}{|l|}{ Polygonaceae } \\
\hline Persicaria decipiens (R.Br.) K.L.Wilson & $\mathrm{t}$ & $?$ & A. Ouédraogo 30 \\
\hline \multicolumn{4}{|l|}{ Pontederiaceae } \\
\hline Eichhornia natans (P.Beauv.) Solms & hy & AA & O. Ouédraogo 206 \\
\hline \multicolumn{4}{|l|}{ RHAMNACEAE } \\
\hline Ziziphus abyssinica A.Rich. & $\mathrm{p}$ & $\mathrm{TA}$ & \\
\hline Ziziphus mucronata Willd. & $\mathrm{p}$ & PRA & \\
\hline Ziziphus spina-christi (L.) Desf. & $\mathrm{p}$ & Paleo & Küppers 1133, Schmidt et al. 6259 \\
\hline \multicolumn{4}{|l|}{ RUBIACEAE } \\
\hline Crossopteryx febrifuga (Afzel. ex G.Don) Benth. & $\mathrm{p}$ & $\mathrm{TA}$ & \\
\hline
\end{tabular}


TABle 2. Continued.

\begin{tabular}{|c|c|c|c|}
\hline FAMILY AND SPECIES & LF & CHOR. & VOUCHER SPECIMENS \\
\hline Fadogia agrestis Schweinf. ex Hiern & c & $\mathrm{S}$ & \\
\hline Fadogia pobeguinii Pobeg. & c & $\mathrm{S}$ & Schmidt 845 \\
\hline Feretia apodanthera Delile & $\mathrm{p}$ & $\mathrm{S}$ & \\
\hline Gardenia aqualla Stapf \& Hutch. & $\mathrm{p}$ & SZ & \\
\hline Gardenia erubescens Stapf \& Hutch. & $\mathrm{p}$ & $\mathrm{S}$ & \\
\hline Gardenia sokotensis Hutch. & $\mathrm{p}$ & $\mathrm{S}$ & \\
\hline Gardenia ternifolia Schumach. \& Thonn. & $\mathrm{p}$ & Pal & \\
\hline Keetia cornelia (Cham. \& Schltdl.) Bridson & $\mathrm{p}$ & GC & O. Ouédraogo 452 \\
\hline Kohautia confusa (Hutch. \& Dalziel) Bremek. & $\mathrm{t}$ & $\mathrm{S}$ & O. Ouédraogo 1, 260, 365 \\
\hline Mitragyna inermis (Willd.) K.Schum. & $\mathrm{p}$ & SZ & Schmidt et al. $6258,6260,6261$ \\
\hline Morelia senegalensis A.Rich. ex DC. & $\mathrm{p}$ & G & O. Ouédraogo 112, 113, 167, 169, Schmidt et al. 6248 \\
\hline Oldenlandia corymbosa L. & $\mathrm{t}$ & Pan & O. Ouédraogo 287 \\
\hline Rytigynia senegalensis Blume & $\mathrm{p}$ & $\mathrm{S}$ & \\
\hline Sarcocephalus latifolius (Sm.) E.A.Bruce & $\mathrm{p}$ & $\mathrm{SZ}$ & \\
\hline Spermacoce chaetocephala DC. & $\mathrm{t}$ & $\mathrm{S}$ & \\
\hline Spermacoce filifolia (Schumach. \& Thonn.) J.-P.Lebrun \& Stork & $\mathrm{t}$ & PRA & \\
\hline Spermacoce hepperana Verdc. & $\mathrm{t}$ & GC & \\
\hline Spermacoce radiata (DC.) Hiern & $\mathrm{t}$ & SZ & \\
\hline Spermacoce ruelliae DC. & $\mathrm{t}$ & $\mathrm{S}$ & \\
\hline Spermacoce stachydea DC. & $\mathrm{t}$ & SZ & O. Ouédraogo 157, 158 \\
\hline \multicolumn{4}{|l|}{ SAPINDACEAE } \\
\hline Allophylus africanus P.Beauv. & $\mathrm{p}$ & $\mathrm{TA}$ & O. Ouédraogo 103 \\
\hline Cardiospermum halicacabum L. & $\mathrm{p}$ & G & O. Ouédraogo 101, 102, 264 \\
\hline Paullinia pinnata L. & $\mathrm{p}$ & AA & Schmidt et al. $6262,6271,6272$ \\
\hline \multicolumn{4}{|l|}{ SAPOTACEAE } \\
\hline Vitellaria paradoxa C.F.Gaertn. & $\mathrm{p}$ & $\mathrm{S}$ & \\
\hline \multicolumn{4}{|l|}{ SCROPHULARIACEAE } \\
\hline Bacopa crenata (P.Beauv.) Hepper & $\mathrm{t}$ & GC & \\
\hline Buchnera hispida Buch.-Ham. ex D.Don & $\mathrm{t}$ & Pal & O. Ouédraogo $48,49,50$ \\
\hline Rhamphicarpa fistulosa (Hochst.) Benth. & c & PRA & O. Ouédraogo 248 \\
\hline Scoparia dulcis L. & $\mathrm{c}$ & PRA & O. Ouédraogo 249 \\
\hline Striga asiatica (L.)Kuntze & $\mathrm{t}$ & Pal & O. Ouédraogo 203 \\
\hline \multicolumn{4}{|l|}{ SOLANACEAE } \\
\hline Physalis angulata $\mathrm{L}$. & $\mathrm{t}$ & Cul & \\
\hline Schwenckia americana L. & $\mathrm{c}$ & $\mathrm{AA}$ & O. Ouédraogo $184,187,345$ \\
\hline \multicolumn{4}{|l|}{ STERCULIACEAE } \\
\hline Cola laurifolia Mast. & $\mathrm{p}$ & GC & Küppers 1141, 1150, Thiombiano et al. 2039 \\
\hline Dombeya quinqueseta (Delile) Exell & $\mathrm{p}$ & TA & Thiombiano et al. 3281, 3283, A. Ouédraogo 292 \\
\hline Melochia corchorifolia L. & hl & Pal & \\
\hline Sterculia setigera Delile & $\mathrm{p}$ & $\mathrm{TA}$ & \\
\hline Waltheria indica $\mathrm{L}$. & c & Pan & \\
\hline \multicolumn{4}{|l|}{ TACCACEAE } \\
\hline Tacca leontopetaloides (L.) Kuntze & $\mathrm{g}$ & Pal & \\
\hline \multicolumn{4}{|l|}{ THYMELAEACEAE } \\
\hline Gnidia kraussiana Meisn. & $\mathrm{c}$ & $\mathrm{S}$ & O. Ouédraogo 461 \\
\hline \multicolumn{4}{|l|}{ Tiliaceae } \\
\hline Corchorus fascicularis Lam. & $\mathrm{t}$ & Pal & \\
\hline Corchorus olitorius L. & $\mathrm{t}$ & Pal & O. Ouédraogo 343 \\
\hline Grewia bicolor Juss. & $\mathrm{p}$ & SZ & Hahn 410 \\
\hline Grewia cissoides Hutch. \& Dalziel & $\mathrm{c}$ & $\mathrm{S}$ & O. Ouédraogo 501 \\
\hline
\end{tabular}


TABLE 2. Continued.

\begin{tabular}{|c|c|c|c|}
\hline FAMILY AND SPECIES & LF & CHOR. & VOUCHER SPECIMENS \\
\hline Grewia flavescens Juss. & $\mathrm{p}$ & Pal & \\
\hline Grewia lasiodiscus K.Schum. & $\mathrm{p}$ & $\mathrm{S}$ & Küppers 1137 \\
\hline Grewia mollis Juss. & $\mathrm{p}$ & PRA & \\
\hline Triumfetta rhomboidea Jacq. & c & Pan & \\
\hline \multicolumn{4}{|l|}{ VERBENACEAE } \\
\hline Gmelina arborea Roxb. & $\mathrm{p}$ & Cul & Küppers 1132 \\
\hline Lantana ukambensis (Vatke) Verdc. & c & SZ & \\
\hline Lippia chevalieri Moldenke & $\mathrm{p}$ & $\mathrm{SZ}$ & \\
\hline Stachytarpheta indica (L.) Vahl & $\mathrm{t}$ & AA & 0. Ouédraogo 2, 3 \\
\hline Vitex chrysocarpa Planch. ex Benth. & $\mathrm{p}$ & $\mathrm{SZ}$ & Küppers 1140, Schmidt et al. 6257, 6264, 6265 \\
\hline Vitex doniana Sweet & $\mathrm{p}$ & $\mathrm{TA}$ & \\
\hline Vitex madiensis Oliv. & $\mathrm{p}$ & $\mathrm{SZ}$ & \\
\hline \multicolumn{4}{|l|}{ VIOLACEAE } \\
\hline Hybanthus enneaspermus (L.) F.Muell. & $\mathrm{t}$ & Pan & O. Ouédraogo 51, 216, 325 \\
\hline \multicolumn{4}{|l|}{ VITACEAE } \\
\hline Ampelocissus leonensis (Hook. f.) Planch. & $\mathrm{p}$ & $\mathrm{S}$ & O. Ouédraogo 322, 326 \\
\hline Cissus cornifolia (Baker) Planch. & c & $\mathrm{S}$ & \\
\hline Cissus populnea Guill. \& Perr. & $\mathrm{p}$ & S & \\
\hline Cissus quadrangularis L. & $\mathrm{p}$ & Pal & 0. Ouédraogo 70 \\
\hline Cissus rufescens Guill. \& Perr. & $\mathrm{p}$ & PRA & \\
\hline Cyphostemma adenocaule (Steud. ex A.Rich.) Desc. ex Wild \& R.B.Drumm. & $\mathrm{p}$ & S & \\
\hline Cyphostemma flavicans (Baker) Desc. & hc & $\mathrm{S}$ & \\
\hline \multicolumn{4}{|l|}{ ZYGOPHYLLACEAE } \\
\hline Balanites aegyptiaca Delile & $\mathrm{p}$ & S & \\
\hline
\end{tabular}

ACKNOWLEDGMENTS: We are grateful for the support of the German Ministry of Education and Research (BMBF) in funding the BIOTA project (funding code 01LC0617D1), to the EU for funding the SUN project (INCO 031685) and UNDESERT project (243906) and the German federal state of Hesse for funding the Biodiversity and Climate Research Institute (BiK-F).

\section{Literature Cited}

Aké Assi, L. 2001. Flore de la Côte d'lvoire: catalogue systématique, biogéographie et écologie. I. Geneva: Editions des Conservatoire et Jardin Botaniques, Boissiera 57.396 p.

Aké Assi, L. 2002. Flore de la Côte d'lvoire: catalogue systématique, biogéographie et écologie. II. Geneva: Editions des Conservatoire et Jardin Botaniques, Boissiera 58. 401 p.

Aké Assi, L., A. Lebbie and E.K. Konan. 2005. La flore des Forêts Classées de la Haute Dodo et du Cavally, Côte d'Ivoire; p. 35-37 In L.E. Alonso, F.Lauginie and G.Rondeau (ed.). A Rapid Biological Assessment of two Classified Forests in South-Western Côte d'Ivoire. Washington, D.C.: Conservation International.

Akoègninou, A., W.J. van der Burg and L.J.G. van der Maesen. 2006. Flore analytique du Bénin. Leiden: Backhuys Publishers. 1034 p.

Arbonnier, M. 2002. Arbres, arbustes et lianes des zones sèches d'Afrique de l'Ouest. Montpellier / Paris: CIRAD, MNHN. 573 p.

Arbonnier, M., N. Barbier, G. De Wispelaere, D. Dulieu, J. Lejoly, A. Mahamane, J.M. Ouadba and M. Saadou. 2002. Cartographie $d u$ couvert végétal du complexe $d u$ « $W »$. Ouagadougou: ECOPAS. 27 p.

Balança G., D. Cornélis and R.Wilson. 2007. Les oiseaux du complexe WAP. Montpellier: CIRAD. 199 p.

Berhaut, J. 1971-1988. Flore illustrée du Sénégal, Vol I-IX. Dakar: Ministère du Développement rural et de l'hydraulique, Direction des Eaux et Fôrets. 4397 p.

Bourlière, F. and M. Hadley. 1970. The ecology of tropical savannas. Annual Review of Ecology and Systematics 1: 125-152.
Brummitt, R.K. 1992. Vascular Plant Families and Genera. Royal Botanic Gardens, Kew: Kew Publishing. 804 p.

Brunken, U., M. Schmidt, S. Dressler, T. Janssen, A. Thombiano and G. Zizka. 2008. West African plants - A Photo Guide. Electronic Database accessible at www.westafricanplants.senckenberg.de Forschungsinstitut Senckenberg, Frankfurt/Main.

Couch, C. and C.T. Williams. 2006. Inventaire botanique rapide de la préfecture de Boké en Guinée; p. 33-37 In H.E. Wright, J. McCullough and M.S. Diallo (ed.). A Rapid Biological Assessment of the Boké Préfecture, Northwestern Guinea. Washington, DC.: Conservation International.

Ekpe, P. 2005. Rapid assessment of Plant Biodiversity of sections of Draw River, Boi-Tano and Krokosua Hills; p. 28-32 In J. McCullough, J.Decher and D.Guba Kpelle (ed.). A biological assessment of the terrestrial ecosystems of the Draw River, Boi-Tano, Tano Nimiri and Krokosua Hills forest reserves, southwestern Ghana. Washington, DC.: Conservation International.

Gautier, L., G.F. Smith, R. Spichiger, R.R. Klopper, S.J. Siebert and C. Chatelain. 2006. Merging tropical and southern African flowering plant data: the African plant database project; p. 629-642 In S.A. Ghazanfar and H.J. Beentje (ed.). Taxonomy and Ecology of African Plants, their conservation and sustainable use-Proceedings of the 17th AETFAT Congress Addis Abeba 21.-26.09.2003. Kew: Kew Publishing.

Gnoumou, A., A. Thiombiano, K. Hahn-Hadjali, B. Abadouabou, M. Sarr and S. Guinko. 2008. Le Parc Urbain Bangr-Wéoogo: une aire de conservation de la diversité floristique au coeur de la ville de Ouagadougou, Burkina Faso. Flora et Vegetatio Sudano-Sambesica 11: 35-48.

Green, A.A. and J.A. Sayer. 1979. The birds of Pendjari and Arli National Parks. Malimbus 1: 14-28.

Green, A.A. 1979. Density Estimate of the Larger Mammals of ArliNational-Park, Upper Volta. Mammalia 43: 59-70.

Guinko, S. 1984. La végétation de la Haute-Volta. Tome 1. PhD thesis, Bordeaux: Université de Bordeaux III. 318 p. 
Guinko, S. and A. Thiombiano. 2005. Florule de la Forêt Classée du Kou (Burkina Faso). Ouagadougou: Université de Ouagadougou. 145 p.

Hahn-Hadjali, K., M. Schmidt and A. Thiombiano. 2006. Phytodiversity dynamics in pastured and protected West African savannas; p. 351359 In S.A. Ghazanfar and H.J. Beentje (ed.), Taxonomy and ecology of African Plants: their conservation and sustainable use - Proceedings of the 17th AETFAT Congress Addis Abeba 21.-26.09.2003. Kew: Kew Publishing.

Holié, J.-L. and N.L. Delamou. 2004. A rapid botanical study of the Forêt Classée du Pic de Fon, Guinea; p. 42-46. In J. McCullough (ed.). A Rapid Biological Assessment of the Forêt Classée du Pic de Fon, Simandou Range, Southeastern Republic of Guinea. Washington, D.C.: Conservation International.

Holié, J.-L. and N.L. Delamou. 2006. Une étude botanique rapide des Forêts Classées de Déré, Diécké et du Mont Béro au sud-est de la Guinée; p. 42-45 In H.E. Wright, J. McCullough, L.E. Alonso, and M.S. Diallo (ed.). A Rapid Biological Assessment of Three Classified Forests in Southeastern Guinea. Washington, DC: Conservation International.

Hutchinson J. and J.M. Dalziel. 1954-1972. Flora of West Tropical Africa. $2^{\text {nd }}$ ed. Vol. I-III. London: Crown Agents for Oversea Governments and Administrations.

Jongkind, C.C.H. 2007a. Rapid survey of the plants of North Lorma, Gola and Grebo National Forests; p. 21-24 In P. Hoke, R. Demey and A. Peal (ed.). A rapid biological assessment of North Lorma, Gola and Grebo National Forests, Liberia. Arlington.: Conservation International.

Jongkind, C.C.H. 2007b. The botanical diversity of the Atewa Range; p. 41-42 In J. McCullough, L.E. Alonso, H.E. Nasrecki, H.E. Wright and Y. Osei-Owusu (ed.). A Rapid Biological Assessment of the Atewa Range Forest Reserve, Eastern Ghana. Arlington: Conservation International.

Jongkind, C.C.H., M. Abu-Juam, M. van Bergen and J. Assi Yapo. 1999. A rapid botanical survey of Parc National de la Marahoué, Côte d'Ivoire; p. 43-45 In T.S. Schulenberg, C.A. Short and P.J. Stephenson (ed.). A biological assessment of Parc National de la Marahoué, Côte d'Ivoire. Arlington: Conservation International.

Koulibaly, A., Goetze, D., Traoré, D. and S. Porembski. 2006. Protected versus exploited savannas: characteristics of the Sudanian vegetation in Ivory Coast. Candollea 61: 425-452

Lamarque F. 2004. Les grands mammifères du complexe WAP. Montpellier: CIRAD. 39 p.

Luke, W.R.Q. 2007. A Brief Botanical Survey at Lokutu; p. 19-20 In: T.M. Butynski and J.McCullough (ed.). A rapid biological assessment of Lokutu, Democratic Republic of Congo. Arlington:Conservation International.

Mbayngone, E., M. Schmidt,K. Hahn-Hadjali, A. Thiombiano and S. Guinko. 2008. Magnoliophyta of the partial faunal reserve of Pama, Burkina Faso. Check List 4(3): 251-266.

Ouattara, T.N. 1994. Diagnostic des potentialités des zones d'extension pour le future. Parc National d'Arly: Rapport de stage ENEF. 22 p.
Ouédraogo, O., A. Thiombiano, K. Hahn-Hadjali and S. Guinko. 2008. Diversité et structure des groupements ligneux du Parc National d'Arly (Est du Burkina Faso). Flora et Vegetatio Sudano-Sambesica 11: 5-16.

Ouédraogo, O., A. Thiombiano, K. Hahn-Hadjali and S. Guinko. 2009. Diversity and dynamics of the juvenile woody vegetation of the Arly National Park (Burkina Faso). Candollea 64: 257-278.

Ouoba, P., A.M. Lykke, I.J. Boussim and S. Guinko. 2006. La flore médicinale de la forêt classée de Niangoloko. Etudes flor. Vég. Burkina Faso 10: 5-16.

Raunkiaer, C. 1905. Types biologiques pour la géographie botanique. Oversigt over Det kongelige Danske Videnskabernes Selskabs Forhandlinger 5: 347-437.

Schmidt, M. 2006. Pflanzenvielfalt in Burkina Faso - Analyse, Modellierung und Dokumentation. PhD thesis accessible at http://publikationen. ub.uni-frankfurt.de/volltexte/2006/3198/. Frankfurt am Main: Goethe-Universität. 188 p.

Schmidt, M., K. König and J.V. Müller. 2008. Modelling species richness and life form composition in Sahelian Burkina Faso with remote sensing data. Journal of Arid Environments 72: 1506-1517.

Schmidt, M., H. Kreft, A. Thiombiano and G. Zizka. 2005. Herbarium collections and field data-based plant diversity maps for Burkina Faso. Diversity and Distributions 11: 509-516.

Schmidt, M., A. Thiombiano, A. Ouédraogo, S. Dressler, K. Hahn-Hadjali and G. Zizka. 2010. Assessment of the flora of Burkina Faso; p. 571576. In X. van der Burgt, J. van der Maesen and J.M. Onana (ed.). Systematics and Conservation of African Plants. Kew: Kew Publishing.

Siaw, D.E.K.A. and J. Dabo. 2007. A rapid botanical survey of the Atewa Range Forest Reserve, Ghana; p. 43-49 In J. McCullough, L.E. Alonso, H.E. Nasrecki, H.E. Wright, and Y. Osei-Owusu (ed.). A Rapid Biological Assessment of the Atewa Range Forest Reserve, Eastern Ghana. Arlington: Conservation International.

Sinsin, B. 2001. Formes de vie et diversité spécifique des associations de forêts claires du nord du Bénin. Systematics and Geography of Plants 71:873-888.

UNEP 2008. Africa: Atlas of our changing environment. Division of early warning and Assessment. Nairobi: UNEP. 374 p.

White, F. 1983. The vegetation of Africa. A descriptive memoir to accompany the Unesco/AETFA/UNSO vegetation map of Africa. Paris: Orstom-Unesco. 356 p.

RECEIVED: April 2010

LAST REVISED: January 2011

ACCEPTED: February 2011

PUBLISHED ONLINE: February 2011

EDITORIAL RESPONSIBILITY: Frederico Augusto Guimarães Guilherme 


\title{
Magnoliophyta, Arly National Park, Tapoa, Burkina Faso
}

\author{
Oumarou Ouédraogo ${ }^{1}$, Marco Schmidt ${ }^{2,3,4^{*}}$, Adjima Thiombiano ${ }^{1}$, Karen Hahn ${ }^{3,4}$, Sita Guinko ${ }^{1}$ and \\ Georg Zizka $2,3,4$
}

1 Université de Ouagadougou, Laboratoire de Biologie et Ecologie Végétales, UFR/SVT. 0309 B.P. 848 Ouagadougou 09, Burkina Faso.

2 Senckenberg Research Institute, Department of Botany and molecular Evolution. Senckenberganlage 25, 60325. Frankfurt am Main, Germany

3 J.W. Goethe-University, Institute for Ecology, Evolution \& Diversity. Siesmayerstr. 70, 60054. Frankfurt am Main, Germany

4 Biodiversity and Climate Research Institute (BiK-F), Senckenberganlage 25, 60325. Frankfurt am Main, Germany.

* Corresponding author. E-mail: marco.schmidt@senckenberg.de

\section{ERRATUM}

In the page 087 there is a mistake in the title (but not in the legend) of Figure 5 and Figure 6, which should be read as:

Figure 5. Chorological spectrum of the flora.

$\square=$ Sudanian; $\square$ = Pantropical; $\square$ = Sudano-zambesian; $\square$ = Paleotropical; - Afrotropical; $\mathbf{\square}=$ Pluriregional African; $\square=$ Sudano-Guinean and Guinean-Congolian; $\square=$ Afro-American; $\square=$ Afro-Malagasy; $\square=$ Cultivated; $\square=$ Cosmopolitan; $\square=$ Unknown.

FIGURE 6. Life form composition: Number of species per life form.

$\square=$ phanerophyte; $\square$ =therophyte; $\square=$ chamaephyte; $\square=$ hemicryptophyte;

$\square=$ geophyte; $\boldsymbol{\square}=$ hydrophyte; $\square=$ helophyte.
We regret these errors.

August 2011. 